\title{
Ergodicity of Stochastic Burgers' System with Dissipative Term
}

\author{
Guoli Zhou, ${ }^{1,2}$ Boling Guo, ${ }^{2}$ Daiwen Huang, ${ }^{2}$ and Yongqian Han ${ }^{2}$ \\ ${ }^{1}$ College of Mathematics and Statistics, Chong Qing University, Chong Qing 401331, China \\ ${ }^{2}$ Institute of Applied Physics and Computational Mathematics, P.O. Box 8009, Beijing 100088, China
}

Correspondence should be addressed to Guoli Zhou; zhouguoli736@126.com

Received 9 October 2013; Revised 6 November 2013; Accepted 6 November 2013

Academic Editor: Hamid Reza Karimi

Copyright (C) 2013 Guoli Zhou et al. This is an open access article distributed under the Creative Commons Attribution License, which permits unrestricted use, distribution, and reproduction in any medium, provided the original work is properly cited.

A 2-dimensional stochastic Burgers equation with dissipative term perturbed by Wiener noise is considered. The aim is to prove the well-posedness, existence, and uniqueness of invariant measure as well as strong law of large numbers and convergence to equilibrium.

\section{Introduction}

The paper is concerned with the 2-dimensional Burgers equation in a bounded domain with Wiener noise as the body forces like this

$$
\begin{gathered}
d u=(v \Delta u+(u \cdot \nabla) u) d t+d W, \quad \text { on }[0, T] \times D, \\
u(t, x)=0, \quad t \in[0, T], x \in \partial D, \\
u(0, x)=u_{0}(x), \quad x \in D
\end{gathered}
$$

where $u(t, x)=\left(u^{1}(t, x), u^{2}(t, x)\right)$ is the velocity field, $v>$ 0 is viscid coefficient, $\Delta$ denotes the Laplace operator, $\nabla$ represents the gradient operator, $W$ stands for the $Q$-Wiener process, and $D$ is a regular bounded open domain of $\mathbb{R}^{2}$. Burgers equation has received an extensive amount of attention since the studies by Burgers in the 1940s (and it has been considered even earlier by Beteman [1] and Forsyth [2]). But it is well known that the Burgers' equation is not a good model for turbulence since it does not perform any chaos. Even if a force is added to equation, all solutions will converge to a unique stationary solution as time goes to infinity. However, if the force is a random one, the result is completely different. So, several authors have indeed suggested to use the stochastic Burgers' equation to model turbulence, see [3-6]. The stochastic equation has also been proposed in [7] to study the dynamics of interfaces.

So far, most of the monographs concerning the equation focus on one-dimensional case, for example, Bertini et al. [8] solved the equation with additive space-time white noise by an adaptation of the Hopf-cole transformation. Da Prato et al. [9] studied the equation via a different approach based on semigroup property for the heat equation on a bounded interval. The more general equation with multiplicative noise was considered by $\mathrm{Da}$ Prato and Debussche [10]. With a similar method, Gyöngy and Nualart [11] extended the Burgers equation from bounded interval to real line. A large deviation principle for the solution was obtained by Gourcy [12]. Concerning the ergodicity, an important paper by Weinan et al. [13] proved that there exists a unique stationary distribution for the solutions of the random inviscid Burgers equation, and typical solutions are piecewise smooth with a finite number of jump discontinuities corresponding to shocks. For model with jumps, Dong and Xu [14] proved that the global existence and uniqueness of the strong, weak, and mild solutions for a one-dimensional Burgers equation perturbed by Lévy noise. When the noise is fractal, Wang et al. [15] get the well-posedness.

The main aim in our paper is to study the large time behavior of stochastic system. There are lots of the literature about the topic (see [16-20]).

Burgers system is a well-known model for mechanics problems. But as far as we know, there are no results about the long-term behavior of stochastic Burgers' system. We think that the difficulty lies in the fact that the dissipative term $\Delta u$ cannot dominate the nonlinear term $(u \cdot \nabla) u$. However, in many practical cases, we cannot ignore the energy dissipation and external forces, especially considering the long-term 
behavior. Therefore, we introduce dissipative term $f(u)$ and study the ergodicity of the following equation:

$$
\begin{gathered}
d u=[\Delta u+(u \cdot \nabla) u-f(u)] d t+d W, \quad \text { on }[0, T] \times D, \\
u(t, x)=0, \quad t \in[0, T], x \in \partial D, \\
u(0, x)=u_{0}(x), \quad x \in D,
\end{gathered}
$$

where $f(u)=\vartheta|u(t, x)|^{2} u(t, x), \vartheta>0,|\cdot|$ denote the absolute value or norm for the real number or two-dimensional vector, respectively.

We believe that our work is new and is worth researching. The methods and results in this paper can be applied to stochastic reaction diffusion equations and stochastic real valued Ginzburg Landau equation in high dimensions. But we cannot extend our result to dynamical systems with statedelays. Since in order to show the existence of an invariant measure, we should consider the segments of a solution. In contrast to the scalar solution process, the process of segments is a Markov process. We show that the process of segments is also Feller and that there exists a solution of which the segments are tight. Then, we apply the Krylov-Bogoliubov method. Since the segment process has values in the infinitedimensional space $C([-r, 0], H)$, boundedness in probability does not automatically imply tightness. For solution processes of infinite-dimensional equations, one often uses compactness of the orbits of the underlying deterministic equation to obtain tightness. For an infinite-dimensional formulation of the functional differential equation, however, such a compactness property does not hold. For ergodicity of stochastic delay equations, we can see [21]. We believe that stochastic Burgers' system with state-delays is a very interesting problem.

In order to study ergodicity of problem (2), we use a remarkable dissipativity property of the stochastic dynamic to obtain the existence of the invariant measure. For uniqueness, we try to use the method from [22] to prove that the distributions $P(t, x, \cdot)$ induced by the solution are equivalent. It is well known that the equivalence of the distributions implies uniqueness, a strong law of large numbers, and the convergence to equilibrium.

The remaining of this paper is organized as follows. Some preliminaries are presented in Section 2, the local existence and global existence are presented, respectively, in Sections 3 and 4 . In Section 5, we obtain the existence and uniqueness of the invariant measure as well as strong law of large numbers, and convergence to equilibrium. As usual, constants $C$ may change from one line to the next; we denote by $C_{a}$ a constant which depends on some parameter $a$.

\section{Preliminaries on the Burgers Equation}

Let $u(t, x)=\left(u^{1}(t, x), u^{2}(t, x)\right)$ be a row vector valued function on $[0, \infty) \times \mathbb{R}^{2}$. And it denotes the following:

$$
|u|^{2}:=\sum_{i=1}^{2}\left|u^{i}\right|^{2}, \quad \partial_{i} u^{j}:=\frac{\partial u^{j}}{\partial x_{i}}, \quad i, j=1,2 .
$$

Let $\left[C^{\infty}(D)\right]^{2}$ be infinitely differentiable 2 -dimensional vector field on $D$, and let $\left[C_{0}^{\infty}(D)\right]^{2}$ be infinitely differentiable 2-dimensional vector field with compact support strictly contained in $D$. We denote by $H^{\alpha}$ the closure of $\left[C^{\infty}(D)\right]^{2}$ in $\left[H^{\alpha}(D)\right]^{2}$, whose norms are denoted by $\|\cdot\|_{H^{\alpha}}$, when $\alpha \neq 0$. Let $H_{0}^{1}, H$ be the closure of $\left[C_{0}^{\infty}(D)\right]^{2}$ in $\left[H^{1}(D)\right]^{2}$ and $\left[L^{2}(D)\right]^{2}$ whose norms are denoted by $\|\cdot\|_{H^{1}}$ and $\|\cdot\|_{H}$, respectively. Without confusion, set $\langle\cdot, \cdot\rangle$ as the inner product in $H$ or $L^{2}(D)$. For $p>0$, let $\|\cdot\|_{L^{p}}$ be the norm of vector filed in Lebesgue spaces $\left[L^{p}(D)\right]^{2} \cdot|\cdot|_{H^{\alpha}}$ represents the norm in the usual sobolev spaces $H^{\alpha}(D)$ for real valued functions on $D$ and $\alpha \in \mathbb{R} ;|\cdot|_{L^{p}}$ stands for the norm in the usual Lebesgue spaces $L^{p}(D)$ for real valued functions on $D$. Denote $A:=-\Delta$; then $A: D(A) \subset H \rightarrow H$ and $D(A)=\left[H^{2}(D)\right]^{2} \cap H_{0}^{1}$. Since $H_{0}^{1}$ coincides with $D\left(A^{1 / 2}\right)$, we can endow $H_{0}^{1}$ with the norm $\|u\|_{H^{1}}=\left\|A^{1 / 2} u\right\|_{H}$. The operator $A$ is positive self-adjoint with compact resolvent; we denote by $0<\alpha_{1} \leq \alpha_{2} \leq \cdots$ the eigenvalues of $A$, and by $e_{1}, e_{2}, \ldots$ the eigenvectors which is a corresponding complete orthonormal system in $H$ satisfying

$$
\begin{array}{ll}
\text { (i) } e_{i} \in\left[C_{0}^{\infty}(D)\right]^{2}, & \\
\text { (ii) }\left|e_{i}(x)\right| \leq C, \quad & \left|\nabla e_{i}(x)\right| \leq C \sqrt{\alpha_{i}}, \\
& x \in D, i=1,2, \ldots,
\end{array}
$$

for some positive constant C. We remark that $\|u\|_{H^{1}}^{2} \geq$ $\alpha_{1}\|u\|_{H}^{2}$. We define the bilinear operator $B(u, v): H^{1} \times H^{1} \rightarrow$ $H^{-1}$ as

$$
\langle B(u, v), z\rangle=\int_{D} z(x) \cdot(u(x) \cdot \nabla) v(x) d x,
$$

for all $z \in H^{1}$. Then, (2) is equivalent to the following abstract equation:

$$
d u(t)+[A u(t)+B(u(t), u(t))+f(u(t))] d t=d W(t) .
$$

$W$ is the $Q$ Wiener process having the following representative:

$$
W(t)=\sum_{n=1}^{\infty} \sqrt{\lambda_{n}} e_{n} \beta_{n}(t), \quad t \in[0, T],
$$

in which $\sum_{n=1}^{\infty} \lambda_{n}<\infty$ and $\beta_{k}$ are a sequence of mutually independent 1-dimensional Brownian motions in a fixed probability space $(\Omega, \mathscr{F}, P)$ adapted to a filtration $\left\{\mathscr{F}_{t}\right\}_{t \geq 0}$.

It can be derived from [23] that the solution to the linear problem corresponding to (2) with the following initial condition:

$$
\begin{gathered}
d u=\Delta u d t+d W, \\
u(t, x)=0, \quad t \in[0, T], x \in \partial D, \\
u(0, x)=u_{0}(x), \quad x \in D,
\end{gathered}
$$

is unique, and when $u_{0}=0$, it has the form of

$$
W_{A}(t)=\int_{0}^{t} e^{(t-s) A} d W(s) .
$$


Let

$$
v(t)=u(t)-W_{A}(t), \quad t \geq 0,
$$

then $u$ is a solution to (2) if and only if it solves the following evolution equation:

$$
\begin{gathered}
\frac{\partial v}{d t}+A v+B\left(v+W_{A}, v+W_{A}\right)+f\left(v+W_{A}\right)=0, \\
v(t, x)=0, \quad t \in[0, T], x \in \partial D, \\
v(0)=u_{0} .
\end{gathered}
$$

So, we see that when $w \in \Omega$ is fixed, this equation is in fact a deterministic equation. From now on, we will study the equation of the form (11) to get the existence and uniqueness of the solution a.s. $w \in \Omega$.

\section{Local Existence in Time}

Definition 1 (see Definition 5.1.1 in [24]). We say a $(\mathscr{F}(t))_{t \geq 0}$ adapted process $v(t)$ is a mild solution to (11), if $v(t) \in$ $C\left([0, T] ; H_{0}^{1}\right)$ and it satisfies

$$
\begin{aligned}
v(t)= & e^{t A} v_{0}+\int_{0}^{t} e^{(t-s) A} B\left(v+W_{A}, v+W_{A}\right) d s \\
& -\int_{0}^{t} e^{(t-s) A} f\left(v+W_{A}\right) d s, \quad t \in[0, T] .
\end{aligned}
$$

Lemma 2. For any $\theta \in(0,1)$, if $\sum_{i=1}^{\infty} \lambda_{i}\left(\alpha_{i}\right)^{\theta}<\infty$, then $A^{1 / 2} W_{A}$ has a version which is $\alpha$-Hölder continuous with respect to $t \in[0, T], x \in D$ with any $\alpha \in] 0, \theta / 2[$.

Proof. Let $T>0$ and $s, t \in[0, T]$; then

$$
\begin{aligned}
E\left|A^{1 / 2} W_{A}(t, x)-A^{1 / 2} W_{A}(s, x)\right|^{2} & \\
= & \sum_{i=1}^{\infty} \lambda_{i} \int_{s}^{t}\left|A^{1 / 2} S(t-\tau) e_{i}(x)\right|^{2} d s \\
& +\sum_{i=1}^{\infty} \lambda_{i} \int_{0}^{s}\left|A^{1 / 2}[S(t-\tau)-S(s-\tau)] e_{i}(x)\right|^{2} d \tau \\
= & : I_{1}(t, s, x)+I_{2}(t, s, x) .
\end{aligned}
$$

Then, we have

$$
\begin{aligned}
& I_{1}(t, s, x) \\
& \quad \leq C \sum_{i=1}^{\infty} \lambda_{i} \alpha_{i} \int_{s}^{t} e^{-2(t-\tau) \alpha_{i}} d \tau \\
& \quad=C \sum_{i=1}^{\infty} \lambda_{i} \alpha_{i}\left(\frac{1-e^{-2(t-s) \alpha_{i}}}{2 \alpha_{i}}\right)
\end{aligned}
$$

$$
\begin{aligned}
& \quad \leq C \sum_{i=1}^{\infty} \lambda_{i}\left(\alpha_{i}\right)^{\theta}|t-s|^{\theta}, \\
& I_{2}(t, s, x) \\
& \quad \leq \frac{1}{2} C \sum_{i=1}^{\infty} \lambda_{i} \alpha_{i} \int_{0}^{s}\left|\left[e^{-(t-\tau) \alpha_{i}}-e^{-(s-\tau) \alpha_{i}}\right]\right|^{2} d \tau \\
& \quad=C \sum_{i=1}^{\infty} \lambda_{i} \alpha_{i} \frac{1}{2 \alpha_{i}}\left[\left(e^{-(t-s) \alpha_{i}}-1\right)^{2}-\left(e^{-t \alpha_{i}}-e^{-s \alpha_{i}}\right)^{2}\right] \\
& \quad \leq C \sum_{i=1}^{\infty} \lambda_{i}\left(\alpha_{i}\right)^{\theta}|t-s|^{\theta} .
\end{aligned}
$$

So, by the estimate of $I_{1}$ and $I_{2}$, we arrive at

$$
E\left|A^{1 / 2} W_{A}(t, x)-A^{1 / 2} W_{A}(s, x)\right|^{2} \leq C \sum_{i=1}^{\infty} \lambda_{i}\left(\alpha_{i}\right)^{\theta}|t-s|^{\theta} .
$$

For $t \in[0, T], x, y \in D$, we get

$$
\begin{aligned}
& E\left|A^{1 / 2} W_{A}(t, x)-A^{1 / 2} W_{A}(t, y)\right|^{2} \\
& \quad=\sum_{i=1}^{\infty} \lambda_{i} \alpha_{i} \int_{0}^{t} e^{-2 \alpha_{i}(t-s)}\left|e_{i}(x)-e_{i}(y)\right|^{2} d s \\
& \quad \leq \sum_{i=1}^{\infty} \lambda_{i}\left|e_{i}(x)-e_{i}(y)\right|^{2} \\
& \quad \leq \sum_{i=1}^{\infty} \lambda_{i}\left(\alpha_{i}\right)^{\theta}|x-y|^{\theta} .
\end{aligned}
$$

Therefore,

$$
\begin{gathered}
E\left|A^{1 / 2} W_{A}(t, x)-A^{1 / 2} W_{A}(s, y)\right|^{2} \\
\leq C\left(|t-s|^{\theta}+|x-y|^{\theta}\right) .
\end{gathered}
$$

As $A^{1 / 2} W_{A}(t, x)-A^{1 / 2} W_{A}(s, y)$ is a Gaussian random variable, we obtain

$$
\begin{gathered}
E\left|A^{1 / 2} W_{A}(t, x)-A^{1 / 2} W_{A}(s, y)\right|^{2 m} \\
\leq C\left(|t-s|^{m \theta}+|x-y|^{m \theta}\right),
\end{gathered}
$$

for $m=1,2, \ldots$ By Kolmogorov' test theorem, we get the conclusion.

Remark 3. An example of the noise satisfying condition of Lemma 2 is

$$
d W(t)=\sum_{n=1}^{\infty} \sqrt{\lambda_{n}} e_{n} d \beta_{n}(t),
$$

where $\left\{\beta_{n}\right\}$ is a sequence of independent 1-dimensional Brownian motion, and $\left\{\lambda_{n}\right\}$ satisfies

$$
\lambda_{n}=n^{-(1+2 \theta)}, \quad \alpha_{n}=n \quad \forall n \in \mathbb{N} .
$$


It is so because the eigenvalues $\alpha_{n}$ of the operator $A$, in 2dimensional space, behave like $n$.

Remark 4. Another example of stochastic noise satisfying Lemma 2 is

$$
A^{-\gamma} \operatorname{LdW}(t)
$$

where $W(t)=\sum_{n=1}^{\infty} e_{n} \beta_{n}(t), L$ is an isomorphism in $H$, and

$$
\gamma \geq \frac{1}{2}+\theta
$$

To prove the local existence of the solution of (1) in sense of Definition 1 , we introduce the space $\mathscr{B}_{m}$ defined by

$$
\mathscr{B}_{m}=\left\{v: v \in C\left(\left[0, T^{*}\right] ; H_{0}^{1}\right),\|v\|_{H^{1}} \leq m, \forall t \in\left[0, T^{*}\right]\right\},
$$

where $T^{*} \geq 0$ which in fact is a stopping time and $m>0$, $p>0$.

Lemma 5. For $u_{0}=\left(u^{1}(0), u^{2}(0)\right),\left\|u_{0}\right\|_{H^{1}}<m$, and $u^{i}(0)$ is adapted to $\mathscr{F}_{0}, i=1,2$; then there exists a unique mild solution $v$ in sense of Definition 1 to (11) in $\mathscr{B}_{m}$.

Proof. Choose a $v$ in $\mathscr{B}_{m}$, and set

$$
\begin{aligned}
\mathscr{L}(v):= & e^{-t A} u_{0} \\
& +\int_{0}^{t} e^{-(t-s) A}\left[\left(v+W_{A}\right) \cdot \nabla\right]\left(v+W_{A}\right) d s \\
& -\int_{0}^{t} e^{-(t-s) A} f\left(v+W_{A}\right) d s .
\end{aligned}
$$

Then,

$$
\begin{aligned}
\|\mathscr{L}(v)\|_{H^{1}} \leq & \left\|e^{-t A} u_{0}\right\|_{H^{1}} \\
& +\left\|\int_{0}^{t} e^{-(t-s) A}\left[\left(v+W_{A}\right) \cdot \nabla\right]\left(v+W_{A}\right) d s\right\|_{H^{1}} \\
& +\left\|\int_{0}^{t} e^{-(t-s) A} f\left(v+W_{A}\right) d s\right\|_{H^{1}}
\end{aligned}
$$

For the second term on the right hand side of (25),

$$
\begin{aligned}
&\left\|e^{-(t-s) A}\left[\left(v+W_{A}\right) \cdot \nabla\right]\left(v+W_{A}\right)\right\|_{H^{1}} \\
&=\left\|e^{-(t-s) A}[u \cdot \nabla] u\right\|_{H^{1}} \\
& \leq \frac{1}{2}\left|e^{-(t-s) A} \partial_{1}\left(u^{1}\right)^{2}\right|_{H^{1}} \\
&+\frac{1}{2}\left|e^{-(t-s) A} \partial_{2}\left(u^{2}\right)^{2}\right|_{H^{1}} \\
&+\left|e^{-(t-s) A} u^{2} \partial_{2} u^{1}\right|_{H^{1}} \\
&+\left|e^{-(t-s) A} u^{1} \partial_{1} u^{2}\right|_{H^{1}} \\
&:= I_{1}+I_{2}+I_{3}+I_{4} .
\end{aligned}
$$

In the following, we will estimate $I_{i}$, respectively, $i=1,2,3,4$. Since $\left\{e^{-t A}\right\}_{t \geq 0}$ is contraction on $L^{p}(D), p \geq 1$, it is known that

$$
\left|e^{-t A} z\right|_{W^{s_{2}, r}} \leq C_{1} t^{\left(s_{1}-s_{2}\right) / 2}|z|_{W^{s_{1}, r}}
$$

for all $z \in W^{s_{1}, r}(D), s_{1}, s_{2} \in \mathbb{R}, s_{1} \leq s_{2}, r \geq 1$, and $C_{1}$ only depends on $s_{1}, s_{2}$, and $r$. Before calculating each $I_{i}$, we outline the Sobolev embedding principle in fractional Sobolev spaces as follows:

$$
W^{\eta_{1}, p_{1}}(D) \subset W^{\eta_{2}, q_{1}}(D)
$$

when

$$
\frac{1}{p_{1}}-\frac{1}{n}\left(\eta_{1}-\eta_{2}\right) \leq \frac{1}{q_{1}} \leq \frac{1}{p_{1}},
$$

where $n$ is the dimension of the spatial. Let $\eta_{1}=3 / 4, p_{1}=$ $2, \eta_{2}=1 / 4, q_{1}=4$ satisfying (29) such that

$$
W^{3 / 4,2}(D) \subset W^{1 / 4,4}(D) \text {. }
$$

For $I_{1}$, by (27) and Theorem A.8 in [25], we get

$$
\begin{aligned}
I_{1} & \leq C_{1}|t-s|^{-7 / 8}\left|\partial_{1}\left(u^{1}\right)^{2}\right|_{H^{-3 / 4}} \\
& =C_{1}|t-s|^{-7 / 8}\left|A^{1 / 8}\left(u^{1}\right)^{2}\right|_{H} \\
& =C_{1}|t-s|^{-7 / 8}\left|2 u^{1} A^{1 / 8} u^{1}+R\right|_{H},
\end{aligned}
$$

where

$$
R=A^{1 / 8}\left(u^{1}\right)^{2}-2 A^{1 / 8} u^{1}
$$

satisfying

$$
|R|_{H} \leq\left|A^{1 / 16} u^{1}\right|_{L^{4}}^{2} \leq\left|u^{1}\right|_{H^{1}}^{2}
$$

The last inequality follows by (30). For the other term added to $R$, we have

$$
\left|2 u^{1} A^{1 / 8} u^{1}\right|_{H} \leq\left|u_{1}\right|_{L^{4}}^{2}+\left|A^{1 / 8} u^{1}\right|_{L^{4}}^{2} \leq 2\left|u^{1}\right|_{H^{1}}^{2}
$$

So, by (31)-(34), we have

$$
I_{1} \leq 3 C_{1}|t-s|^{-7 / 8}\left|u^{1}\right|_{H^{1}}^{2} .
$$

Similarly, we get for $I_{2}$ that

$$
I_{2} \leq 3 C_{1}|t-s|^{-7 / 8}\left|u^{2}\right|_{H^{1}}^{2} .
$$

For $I_{3}$, by Theorem A.8 in [25], we get

$$
\begin{aligned}
& I_{3} \leq\left|e^{-(t-s) A} u^{2} A^{1 / 2} u^{1}\right|_{H^{1}} \\
& =\left|e^{-(t-s) A}\left[A^{1 / 4}\left(u^{2} A^{1 / 4} u^{1}\right)-\left(A^{1 / 4} u^{1}\right)\left(A^{1 / 4} u^{2}\right)-R_{1}\right]\right|_{H^{1}},
\end{aligned}
$$


where

$$
R_{1}=A^{1 / 4}\left(u^{2} A^{1 / 4} u^{1}\right)-\left[A^{1 / 4} u^{2}\right]\left[A^{1 / 4} u^{1}\right]-u^{2} A^{1 / 2} u^{1} .
$$

For $R_{1}$, we have

$$
\begin{aligned}
\mid e^{-(t-s) A} & \left.R_{1}\right|_{H^{1}} \\
& \leq C_{1}|t-s|^{-1 / 2}\left|R_{1}\right|_{H} \\
& \leq C_{1}|t-s|^{-1 / 2}\left|A^{1 / 4} u^{1}\right|_{L^{4}} \cdot\left|A^{1 / 4} u^{2}\right|_{L^{4}} \\
& \leq C_{1}|t-s|^{-1 / 2}\left(\left|u^{1}\right|_{H^{1}}^{2}+\left|u^{2}\right|_{H^{1}}^{2}\right) .
\end{aligned}
$$

For the first term on the right hand side of (37), by (27), we have

$$
\begin{aligned}
& \left|e^{-(t-s) A} A^{1 / 4}\left(u^{2} A^{1 / 4} u^{1}\right)\right|_{H^{1}} \\
& \quad=\left|e^{-(t-s) A} A^{3 / 4}\left(u^{2} A^{1 / 4} u^{1}\right)\right|_{H} \\
& \quad \leq C_{1}|t-s|^{-3 / 4}\left|u^{2} A^{1 / 4} u^{1}\right|_{H} \\
& \quad \leq C_{1}|t-s|^{-3 / 4}\left(\left|u^{2}\right|_{L^{4}}^{2}+\left|A^{1 / 4} u^{1}\right|_{L^{4}}^{2}\right) \\
& \quad \leq C_{1}|t-s|^{-3 / 4}\left(\left|u^{2}\right|_{H^{1}}^{2}+\left|u^{1}\right|_{H^{1}}^{2}\right) .
\end{aligned}
$$

For the second term on the right hand side of (37), by (27), we obtain

$$
\begin{aligned}
\mid e^{-(t-s) A} & {\left.\left[A^{1 / 4} u^{2} \cdot A^{1 / 4} u^{1}\right]\right|_{H^{1}} } \\
& \leq C_{1}|t-s|^{-1 / 2}\left|A^{1 / 4} u^{2} \cdot A^{1 / 4} u^{1}\right|_{H} \\
& \leq C_{1}|t-s|^{-1 / 2}\left(\left|u^{1}\right|_{H^{1}}^{2}+\left|u^{2}\right|_{H^{1}}^{2}\right) .
\end{aligned}
$$

From (37) to (41), we get for $I_{3}$ that

$$
I_{3} \leq C\left(|t-s|^{-1 / 2}+|t-s|^{-3 / 4}\right)\left(\left|u^{1}\right|_{H^{1}}^{2}+\left|u^{2}\right|_{H^{1}}^{2}\right) .
$$

Analogously, for $I_{4}$, we get

$$
I_{4} \leq C\left(|t-s|^{-1 / 2}+|t-s|^{-3 / 4}\right)\left(\left|u^{1}\right|_{H^{1}}^{2}+\left|u^{2}\right|_{H^{1}}^{2}\right) .
$$

By (26), (35), (36), (42), and (43), we have

$$
\begin{aligned}
\| e^{-(t-s) A} & {\left[\left(v+W_{A}\right) \cdot \nabla\right]\left(v+W_{A}\right) \|_{H^{1}} } \\
\leq & C\left(|t-s|^{-1 / 2}+|t-s|^{-3 / 4}+|t-s|^{-7 / 8}\right) \\
& \times\left(\left|u^{1}\right|_{H^{1}}^{2}+\left|u^{2}\right|_{H^{1}}^{2}\right) .
\end{aligned}
$$

As $u=v+W_{A}$, by (44), for $t \leq T^{*}$, we have

$$
\begin{aligned}
& \int_{0}^{t} d s\left\|e^{-(t-s) A}\left[\left(v+W_{A}\right) \cdot \nabla\right]\left(v+W_{A}\right)\right\|_{H^{1}} \\
& \quad \leq C\left(t^{1 / 8}+t^{1 / 4}+t^{1 / 2}\right)\left(\sup _{t \in\left[0, T^{*}\right]}\|v\|_{H^{1}}^{2}+\sup _{t \in[0, T]}\left\|W_{A}\right\|_{H^{1}}^{2}\right) .
\end{aligned}
$$

Since by Lemma 2,

$$
\sup _{t \in[0, T]}\left\|W_{A}\right\|_{H^{1}}^{2}<\infty .
$$

For the last term on the right hand side of (25), we have

$$
\begin{aligned}
& \left\|e^{-(t-s) A} f\left(v+W_{A}\right)\right\|_{H^{1}} \\
& \quad \leq C|t-s|^{-1 / 2}\left(\left\|v+W_{A}\right\|_{L^{6}}^{3}\right) \\
& \quad \leq C|t-s|^{-1 / 2}\left(\left\|W_{A}\right\|_{H^{1}}^{3}+\|v\|_{H^{1}}^{3}\right) .
\end{aligned}
$$

Therefore,

$$
\begin{gathered}
\left\|\int_{0}^{t} e^{-(t-s) A} f\left(v+W_{A}\right) d s\right\|_{H^{1}} \\
\quad \leq C\left(1+m^{3}\right) \int_{0}^{t}|t-s|^{-1 / 2} d s \\
\quad \leq C\left(1+m^{3}\right) T^{* 1 / 2} .
\end{gathered}
$$

So by (25), (45), and (48), when $T^{*}$ is small enough,

$$
\|\mathscr{L}(v)\|_{H^{1}} \leq m .
$$

For each $v_{1}, v_{2} \in \mathscr{B}_{m}$, set $u_{i}=v_{i}+W_{A}, i=1,2$. To simplify the notation in the following calculation, we denote $u_{i}=\left(u_{i}^{1}, u_{i}^{2}\right)$, $i=1,2$. Then,

$$
\begin{aligned}
\mathscr{L}\left(v_{1}\right) & -\mathscr{L}\left(v_{2}\right) \\
= & \int_{0}^{t} e^{-(t-s) A}\left[\left(u_{1} \cdot \nabla\right) u_{1}-\left(u_{2} \cdot \nabla\right) u_{2}\right] d s \\
& +\int_{0}^{t} e^{-(t-s) A}\left[f\left(u_{1}\right)-f\left(u_{2}\right)\right] d s .
\end{aligned}
$$

So,

$$
\begin{aligned}
\| \mathscr{L}\left(v_{1}\right) & -\mathscr{L}\left(v_{2}\right) \|_{H^{1}} \\
\leq & \int_{0}^{t}\left\|e^{-(t-s) A}\left[\left(u_{1} \cdot \nabla\right) u_{1}-\left(u_{2} \cdot \nabla\right) u_{2}\right]\right\|_{H^{1}} d s \\
& +\int_{0}^{t}\left\|e^{-(t-s) A}\left[f\left(u_{1}\right)-f\left(u_{2}\right)\right]\right\|_{H^{1}} d s .
\end{aligned}
$$

In order to simplify the notation, we set

$$
\left(u_{1} \cdot \nabla\right) u_{1}-\left(u_{2} \cdot \nabla\right) u_{2}=\left(f_{1}+f_{2}, f_{3}+f_{4}\right),
$$

where

$$
\begin{gathered}
f_{1}=\frac{1}{2} \partial_{1}\left[\left(u_{1}^{1}\right)^{2}-\left(u_{2}^{1}\right)^{2}\right], \\
f_{2}=u_{1}^{2} \partial_{2} u_{1}^{1}-u_{2}^{2} \partial_{2} u_{2}^{1}, \\
f_{3}=\frac{1}{2} \partial_{2}\left[\left(u_{1}^{2}\right)^{2}-\left(u_{2}^{2}\right)^{2}\right], \\
f_{4}=u_{1}^{1} \partial_{1} u_{1}^{2}-u_{2}^{1} \partial_{1} u_{2}^{2} .
\end{gathered}
$$


Then, we estimate $f_{i}, i=1,2,3,4$, respectively. For $f_{1}$, we have

$$
\begin{aligned}
\mid e^{-(t-s) A} & \left.f_{1}\right|_{H^{1}} \\
= & \left|e^{-(t-s) A} A^{1 / 2}\left[\left(u_{1}^{1}-u_{2}^{1}\right)\left(u_{1}^{1}+u_{2}^{1}\right)\right]\right|_{H^{1}} \\
\leq & C|t-s|^{-7 / 8}\left|A^{1 / 8}\left[\left(u_{1}^{1}\right)^{2}-\left(u_{2}^{1}\right)^{2}\right]\right|_{H} \\
= & C|t-s|^{-7 / 8}\left|A^{1 / 8}\left[\left(u_{1}^{1}-u_{1}^{2}\right)\left(u_{1}^{1}+u_{1}^{2}\right)\right]\right|_{H} \\
= & C|t-s|^{-7 / 8}\left|\left[A^{1 / 8}\left(u_{1}^{1}-u_{1}^{2}\right)\right]\left(u_{1}^{1}+u_{1}^{2}\right)\right|_{H} \\
& +C|t-s|^{-7 / 8}\left|\left[A^{1 / 8}\left(u_{1}^{1}+u_{1}^{2}\right)\right]\left(u_{1}^{1}-u_{1}^{2}\right)+R_{2}\right|_{H} .
\end{aligned}
$$

We first consider

$$
\begin{aligned}
\left|R_{2}\right|_{H} & \leq C\left|A^{1 / 16}\left(u_{1}^{1}-u_{2}^{1}\right)\right|_{L^{4}} \cdot\left|A^{1 / 16}\left(u_{1}^{1}+u_{2}^{1}\right)\right|_{L^{4}} \\
& \leq C\left|u_{1}^{1}-u_{2}^{1}\right|_{H^{1}} \cdot\left|u_{1}^{1}+u_{2}^{1}\right|_{H^{1}} .
\end{aligned}
$$

For the other term added to $R_{2}$,

$$
\begin{aligned}
& \quad\left|\left[A^{1 / 8}\left(u_{1}^{1}+u_{2}^{1}\right)\right]\left(u_{1}^{1}-u_{2}^{1}\right)\right|_{H} \\
& \quad \leq\left|u_{1}^{1}+u_{2}^{1}\right|_{H^{1}} \cdot\left|u_{1}^{1}-u_{2}^{1}\right|_{H^{1}} .
\end{aligned}
$$

By (54)-(56),

$$
\left|e^{-(t-s) A} f_{1}\right|_{H^{1}} \leq C|t-s|^{-7 / 8}\left|u_{1}^{1}-u_{2}^{1}\right|_{H^{1}} \cdot\left|u_{1}^{1}+u_{2}^{1}\right|_{H^{1}} .
$$

Analogously, for $f_{3}$,

$$
\left|e^{-(t-s) A} f_{3}\right|_{H^{1}} \leq C|t-s|^{-7 / 8}\left|u_{1}^{1}-u_{2}^{1}\right|_{H^{1}} \cdot\left|u_{1}^{1}+u_{2}^{1}\right|_{H^{1}} .
$$

For $f_{2}$, by (53), we have

$$
\begin{aligned}
\left|e^{-(t-s) A} f_{2}\right|_{H^{1}}= & \left|e^{-(t-s) A}\left(u_{1}^{2} \partial_{2} u_{1}^{1}-u_{2}^{2} \partial_{2} u_{2}^{1}\right)\right|_{H^{1}} \\
\leq & \left|e^{-(t-s) A}\left(u_{1}^{2}\left(\partial_{2} u_{1}^{1}-\partial_{2} u_{2}^{1}\right)\right)\right|_{H^{1}} \\
& +\left|e^{-(t-s) A}\left(\left(u_{1}^{2}-u_{2}^{2}\right) \partial_{2} u_{2}^{1}\right)\right|_{H^{1}} .
\end{aligned}
$$

For the first term on the right hand side of (59), we have

$$
\begin{aligned}
\mid e^{-(t-s) A} & \left.\left(u_{1}^{2}\left(\partial_{2} u_{1}^{1}-\partial_{2} u_{2}^{1}\right)\right)\right|_{H^{1}} \\
\leq & \left|e^{-(t-s) A}\left(u_{1}^{2} A^{1 / 2}\left(u_{1}^{1}-u_{2}^{1}\right)\right)\right|_{H^{1}} \\
=\mid e^{-(t-s) A} & \left\{A^{1 / 4}\left[u_{1}^{2} A^{1 / 4}\left(u_{1}^{1}-u_{2}^{1}\right)\right]\right. \\
& \left.-\left[A^{1 / 4} u_{1}^{2}, A^{1 / 4}\left(u_{1}^{1}-u_{2}^{1}\right)\right]-R_{3}\right\}\left.\right|_{H^{1}} .
\end{aligned}
$$

For $R_{3}$,

$$
\begin{aligned}
& \left|e^{-(t-s) A} R_{3}\right|_{H^{1}} \\
& \quad \leq|t-s|^{-1 / 2}\left|R_{3}\right|_{H} \\
& \quad \leq|t-s|^{-1 / 2}\left|A^{1 / 4} u_{1}^{2}\right|_{L^{4}} \cdot\left|A^{1 / 4}\left(u_{1}^{1}-u_{2}^{1}\right)\right|_{L^{4}} \\
& \quad=|t-s|^{-1 / 2}\left|u_{1}^{2}\right|_{H^{1}} \cdot\left|u_{1}^{1}-u_{2}^{1}\right|_{H^{1}} .
\end{aligned}
$$

For the first term on the right hand side of (60), we arrive at

$$
\begin{aligned}
\left|e^{-(t-s) A} A^{1 / 4}\left[u_{1}^{2} A^{1 / 4}\left(u_{1}^{1}-u_{2}^{1}\right)\right]\right|_{H^{1}} \\
\quad=\left|e^{-(t-s) A} A^{3 / 4}\left[u_{1}^{2} A^{1 / 4}\left(u_{1}^{1}-u_{2}^{1}\right)\right]\right|_{H} \\
\quad \leq|t-s|^{-3 / 4}\left|u_{1}^{2} A^{1 / 4}\left(u_{1}^{1}-u_{2}^{1}\right)\right|_{H} \\
\quad \leq|t-s|^{-3 / 4}\left|u_{1}^{2}\right|_{L^{4}} \cdot\left|A^{1 / 4}\left(u_{1}^{1}-u_{2}^{1}\right)\right|_{L^{4}} \\
\leq|t-s|^{-3 / 4}\left|u_{1}^{2}\right|_{H^{1}} \cdot\left|u_{1}^{1}-u_{2}^{1}\right|_{H^{1}} .
\end{aligned}
$$

For the second term on the right hand side of (60), we obtain

$$
\begin{aligned}
& \left|e^{-(t-s) A}\left[\left(A^{1 / 4} u_{1}^{2}\right)\left(A^{1 / 4}\left(u_{1}^{1}-u_{2}^{1}\right)\right)\right]\right|_{H^{1}} \\
& \leq|t-s|^{-1 / 2}\left|\left[A^{1 / 4} u_{1}^{2}\right] \cdot\left[A^{1 / 4}\left(u_{1}^{1}-u_{2}^{1}\right)\right]\right|_{H} \\
& \leq|t-s|^{-1 / 2}\left|A^{1 / 4} u_{1}^{2}\right|_{L^{4}} \cdot\left|A^{1 / 4}\left(u_{1}^{1}-u_{2}^{1}\right)\right|_{L^{4}} \\
& \leq|t-s|^{-1 / 2}\left|u_{1}^{2}\right|_{H^{1}} \cdot\left|u_{1}^{1}-u_{2}^{1}\right|_{H^{1}} .
\end{aligned}
$$

By (59)-(63), we get for $f_{2}$ that

$$
\begin{aligned}
\mid e^{(t-s) A} & \left.f_{2}\right|_{H^{1}} \\
\leq & C\left(|t-s|^{-1 / 2}+|t-s|^{-3 / 4}\right) \\
& \times\left(\left\|u_{1}\right\|_{H^{1}}+\left\|u_{2}\right\|_{H^{1}}\right)\left\|u_{1}-u_{2}\right\|_{H^{1}} .
\end{aligned}
$$

Similarly, we get for $f_{4}$ that

$$
\begin{aligned}
\mid e^{(t-s) A} & \left.f_{4}\right|_{H^{1}} \\
\leq & C\left(|t-s|^{-1 / 2}+|t-s|^{-3 / 4}\right) \\
& \times\left(\left\|u_{1}\right\|_{H^{1}}+\left\|u_{2}\right\|_{H^{1}}\right)\left\|u_{1}-u_{2}\right\|_{H^{1}} .
\end{aligned}
$$

By (52), (53), (57), (58), (64), and (65), we have

$$
\begin{aligned}
\| e^{-(t-s) A} & {\left[\left(u_{1} \cdot \nabla\right) u_{1}-\left(u_{2} \cdot \nabla\right) u_{2}\right] \|_{H^{1}} } \\
\leq & \sum_{i=1}^{4}\left|e^{-(t-s) A} f_{i}\right|_{H^{1}} \\
\leq & C\left(|t-s|^{-1 / 2}+|t-s|^{-3 / 4}+|t-s|^{-7 / 8}\right) \\
& \times\left(\left\|u_{1}\right\|_{H^{1}}+\left\|u_{2}\right\|_{H^{1}}\right)\left\|u_{1}-u_{2}\right\|_{H^{1}} \\
\leq & C(2 m+1)\left(|t-s|^{-1 / 2}+|t-s|^{-3 / 4}+|t-s|^{-7 / 8}\right) \\
& \times\left\|v_{1}-v_{2}\right\|_{H^{1}} .
\end{aligned}
$$

For the second term on the right hand side of (51), we have

$$
f\left(u_{1}\right)-f\left(u_{2}\right)=\left(h_{1}, h_{2}\right),
$$

where

$$
\begin{aligned}
& h_{1}=\left|u_{1}\right|^{2} u_{1}^{1}-\left|u_{2}\right|^{2} u_{2}^{1}, \\
& h_{2}=\left|u_{1}\right|^{2} u_{1}^{2}-\left|u_{2}\right|^{2} u_{2}^{2} .
\end{aligned}
$$


Then,

$$
\begin{aligned}
\left|e^{(t-s) A} h_{1}\right|_{H^{1}} \\
\leq\left. C|t-s|^{-1 / 2}|| u_{1}\right|^{2} u_{1}^{1}-\left.\left|u_{2}\right|^{2} u_{2}^{1}\right|_{H} \\
\leq\left. C|t-s|^{-1 / 2}|| u_{1}\right|^{2} u_{1}^{1}-\left.\left|u_{2}\right|^{2} u_{1}^{1}\right|_{H} \\
\quad+\left.C|t-s|^{-1 / 2}|| u_{2}\right|^{2} u_{1}^{1}-\left.\left|u_{2}\right|^{2} u_{2}^{1}\right|_{H} \\
\leq C|t-s|^{-1 / 2}|| v_{1}-v_{2}\left|\cdot\left(\left|v_{1}\right|+\left|v_{2}\right|+2\left|W_{A}\right|\right) \cdot\right| u_{1}^{1}||_{H} \\
\quad+\left.C|t-s|^{-1 / 2}|| u_{2}\right|^{2} \cdot \mid v_{1}^{1}-v_{2}^{1} \|_{H} \\
\leq C|t-s|^{-1 / 2}\left\|v_{1}-v_{2}\right\|_{L^{4}} \cdot\left|\left(\left|v_{1}\right|+\left|v_{2}\right|+2\left|W_{A}\right|\right) \cdot\right| u_{1}^{1} \|_{L^{4}} \\
\quad+C|t-s|^{-1 / 2}\left\|v_{1}-v_{2}\right\|_{L^{4}} \cdot\left\|u_{2}\right\|_{L^{4}}^{2} \\
\leq C|t-s|^{-1 / 2}\left\|v_{1}-v_{2}\right\|_{L^{4}}\left(\left\|v_{1}\right\|_{L^{8}}^{2}+\left\|v_{2}\right\|_{L^{8}}^{2}+1\right) \\
\leq C|t-s|^{-1 / 2}\left\|v_{1}-v_{2}\right\|_{H^{1}}\left(\left\|v_{1}\right\|_{H^{1}}^{2}+\left\|v_{2}\right\|_{H^{1}}^{2}+1\right) .
\end{aligned}
$$

Similarly, we can get the same estimate for $h_{2}$. So, we have

$$
\begin{aligned}
& \int_{0}^{t}\left\|e^{(t-s) A}\left[f\left(u_{1}\right)-f\left(u_{2}\right)\right]\right\|_{H^{1}} d s \\
& \quad \leq C\left(1+m^{2}\right) T^{* 1 / 2} \sup _{t \in\left[0, T^{*}\right]}\left\|v_{1}(t)-v_{2}(t)\right\|_{H^{1}} .
\end{aligned}
$$

By (51), (66), and (70), we have

$$
\begin{aligned}
& \left\|\mathscr{L}\left(v_{1}\right)-\mathscr{L}\left(v_{2}\right)\right\|_{H^{1}} \\
& \quad \leq C\left[T^{* 1 / 2}+T^{* 1 / 4}+T^{* 1 / 8}\right] \cdot\left(\sup _{t \in\left[0, T^{*}\right]}\left\|v_{1}-v_{2}\right\|_{H^{1}}\right) .
\end{aligned}
$$

By (49), (71), and fixed point principle, we get the conclusion.

Remark 6. By making some minor modifications in the proof of Lemma 5, we can see that the conclusion in Lemma 5 is also true for (1). Our original aim is to get the global wellposedness of (1), but we find that the dissipative term $\Delta u$ cannot dominate the nonlinear term $(u \cdot \nabla) u$. So, we introduce the dissipative term $|u|^{2} u$ which will also play an important role in obtaining the ergodicity.

\section{Global Existence}

Theorem 7. With conditions in Lemma 2, for $v \in C([0, T]$; $H_{0}^{1}$ ) satisfying (12), when $9>1 / 16$, one has

$$
\|v\|_{H^{1}} \leq\left(C_{T}+\left\|v_{0}\right\|_{H^{1}}^{2}\right) e^{C_{T}} .
$$

Subsequently, one gets the existence of the global solution belonging to $C\left([0, T] ; H_{0}^{1}\right)$.
Proof. Let $\left\{u_{n}^{0}\right\}_{n \geq 1}$ be a sequence of vectors which satisfies $u_{n}^{0}=\left(u_{n}^{0,1}, u_{n}^{0,2}\right)$ and $u_{n}^{0, i} \in C_{0}^{\infty}(D), i=1,2, n \geq 1$, such that

$$
u_{n}^{0} \longrightarrow u_{0}, \quad \text { as } n \longrightarrow \infty,
$$

in sense of $\|\cdot\|_{H^{1}}$. Let $\left\{W_{n}\right\}_{n \geq 1}$ be a sequence of regular process, such that

$$
\begin{array}{r}
A^{a / 2} W_{A}^{n}:=A^{a / 2} \int_{0}^{t} e^{(t-s) A} d W_{n}(s) \longrightarrow A^{a / 2} W_{A}(t), \\
\text { as } n \rightarrow \infty,
\end{array}
$$

in $C(T \times D)$ when $a=0$ or $a=1$. For $h=\left(h_{1}, h_{2}\right)$, $h_{i} \in C([0, T] \times D ; \mathbb{R}),\|h\|_{C(T \times D)}:=\sum_{i=1}^{2}\left|h_{i}\right|_{C(T \times D)}$, where $\left|h_{i}\right|_{C(T \times D)}=\sup _{(t, x) \in[0, T] \times D}\left|h_{i}\right|$. Then, by $(74)$, we have

$$
\begin{gathered}
\sup _{\{n \geq 1\}}\left\|W_{A}^{n}\right\|_{C(T \times D)}<\infty, \\
\sup _{\{n \geq 1\}} \sup _{t \in[0, T]}\left|A^{1 / 2} W_{A}^{n}\right|<\infty .
\end{gathered}
$$

If $v_{n}$ satisfies

$$
\begin{aligned}
v_{n}= & e^{t A} u_{n}^{0}+\int_{0}^{t} e^{(t-s) A}\left[\left(v_{n}+W_{A}\right) \cdot \nabla\right]\left(v_{n}+W_{A}\right) d s \\
& -\int_{0}^{t} e^{(t-s) A} f\left(v_{n}+W_{A}\right),
\end{aligned}
$$

then, $v_{n}$ is regular, such that

$$
\frac{\partial v_{n}}{\partial t}+A v_{n}+B\left(v_{n}+W_{A}^{n}, v_{n}+W_{A}^{n}\right)+f\left(v_{n}+W_{A}^{n}\right)=0 .
$$

Taking inner product with respect to $v_{n}$ in (78), we have

$$
\begin{aligned}
\left\langle\frac{\partial v_{n}}{\partial t},\right. & \left.v_{n}\right\rangle+\left\langle A v_{n}, v_{n}\right\rangle \\
& +\left\langle B\left(v_{n}+W_{A}^{n}, v_{n}+W_{A}^{n}\right), v_{n}\right\rangle \\
& +\left\langle f\left(v_{n}+W_{A}^{n}\right), v_{n}\right\rangle=0 .
\end{aligned}
$$

For simplicity, we calculate the third term on the left hand side of (79) first as follows:

$$
\begin{aligned}
\left\langleB \left( v_{n}+\right.\right. & \left.\left.W_{A}^{n}, v_{n}+W_{A}^{n}\right), v_{n}\right\rangle \\
= & \left\langle\left(v_{n}^{1}+W_{A, 1}^{n}\right) \partial_{1}\left(v_{n}^{1}+W_{A, 1}^{n}\right), v_{n}^{1}\right\rangle \\
& +\left\langle\left(v_{n}^{2}+W_{A, 2}^{n}\right) \partial_{2}\left(v_{n}^{1}+W_{A, 1}^{n}\right), v_{n}^{1}\right\rangle \\
& +\left\langle\left(v_{n}^{1}+W_{A, 1}^{n}\right) \partial_{1}\left(v_{n}^{2}+W_{A, 2}^{n}\right), v_{2}^{n}\right\rangle \\
& +\left\langle\left(v_{n}^{2}+W_{A, 2}^{n}\right) \partial_{2}\left(v_{n}^{2}+W_{A, 2}^{n}\right), v_{n}^{2}\right\rangle \\
= & I_{1}+I_{2}+I_{3}+I_{4},
\end{aligned}
$$


where $W_{A}^{n}=\left(W_{A, 1}^{n}, W_{A, 2}^{n}\right)$. For $I_{1}$, we have

$$
\begin{aligned}
I_{1}= & \left\langle\left(v_{n}^{1}+W_{A, 1}^{n}\right) \partial_{1}\left(v_{n}^{1}+W_{A, 1}^{n}\right), v_{n}^{1}\right\rangle \\
= & \left\langle v_{n}^{1} \partial_{1} v_{n}^{1}, v_{n}^{1}\right\rangle+\left\langle W_{A, 1}^{n} \partial_{1} v_{n}^{1}, v_{n}^{1}\right\rangle \\
& +\left\langle v_{n}^{1} \partial_{1} W_{A, 1}^{n}, v_{n}^{1}\right\rangle+\left\langle W_{A, 1}^{n} \partial_{1} W_{A, 1}^{n}, v_{n}^{1}\right\rangle .
\end{aligned}
$$

In the following, we estimate the four terms for $I_{1}$, respectively. For the first term,

$$
\begin{aligned}
\left\langle v_{n}^{1} \partial_{1} v_{n}^{1}, v_{n}^{1}\right\rangle & =\int_{D}\left(v_{n}^{1}\right)^{2} \partial_{1} v_{n}^{1} d x \\
& =\int_{D} \partial_{1}\left[\frac{\left(v_{n}^{1}\right)^{3}}{3}\right] d x=0 .
\end{aligned}
$$

For the second term, by (75), we have

$$
\begin{aligned}
& \left\langle W_{A, 1}^{n} \partial_{1} v_{n}^{1}, v_{n}^{1}\right\rangle \\
& \leq C\left|v_{n}^{1}\right|_{H}^{2}+\varepsilon \int_{D}\left(\partial_{1} v_{n}^{1}\right)^{2} d x \\
& \leq C\left|v_{n}^{1}\right|_{H}^{2}+\varepsilon\left|v_{n}^{1}\right|_{H^{1}}^{2} .
\end{aligned}
$$

similarly, for the third term,

$$
\begin{aligned}
& \left|\left\langle v_{n}^{1} \partial_{1} W_{A, 1}^{n}, v_{n}^{1}\right\rangle\right| \\
& =\left|\int_{D}\left(v_{n}^{1}\right)^{2} \partial_{1} W_{A, 1}^{n} d x\right| \\
& =\left|\int_{D} W_{A, 1}^{n} \partial_{1}\left(v_{n}^{1}\right)^{2} d x\right| \\
& \leq C\left|\int_{D} v_{n}^{1} \partial_{1} v_{n}^{1} d x\right| \\
& \leq C\left|v_{n}^{1}\right|_{H}^{2}+\varepsilon\left|v_{n}^{1}\right|_{H^{1}}^{2} .
\end{aligned}
$$

For the last term, by (75) and (76),

$$
\begin{gathered}
\left|\left\langle W_{A, 1}^{n} \partial_{1} W_{A, 1}^{n}, v_{n}^{1}\right\rangle\right| \\
\leq C\left|\int_{D} \partial_{1} v_{n}^{1} d x\right| \\
\leq C+\varepsilon\left|v_{n}^{1}\right|_{H^{1}}^{2} .
\end{gathered}
$$

By (81)-(85), it follows that

$$
I_{1} \leq C\left(1+\left\|v_{n}\right\|_{H}^{2}\right)+4 \varepsilon\left\|v_{n}\right\|_{H^{1}}^{2} .
$$

Similarly,

$$
I_{4} \leq C\left(1+\left\|v_{n}\right\|_{H}^{2}\right)+4 \varepsilon\left\|v_{n}\right\|_{H^{1}}^{2} .
$$

For $I_{3}$,

$$
\begin{aligned}
I_{3}= & \left\langle v_{n}^{1} \partial_{1} v_{n}^{2}, v_{n}^{2}\right\rangle+\left\langle v_{n}^{1} \partial_{1} W_{A, 2}^{n}, v_{n}^{2}\right\rangle \\
& +\left\langle W_{A, 1}^{n} \partial_{1} v_{n}^{2}, v_{n}^{2}\right\rangle+\left\langle W_{A, 1}^{n} \partial_{1} W_{A, 2}^{n}, v_{n}^{2}\right\rangle .
\end{aligned}
$$

For the first term on the right hand side of (88), we deduce that

$$
\begin{aligned}
\left|\left\langle v_{n}^{1} \partial_{1} v_{n}^{2}, v_{n}^{2}\right\rangle\right| & =\frac{1}{2}\left|\int_{D} v_{n}^{1} \partial_{1}\left(v_{n}^{2}\right)^{2} d x\right| \\
& =\frac{1}{2}\left|\int_{D} \partial_{1} v_{n}^{1} \cdot\left(v_{n}^{2}\right)^{2} d x\right| \\
& \leq \frac{1}{2}\left|v_{n}^{2}\right|_{L^{4}}^{2} \cdot\left|v_{n}^{1}\right|_{H^{1}} \\
& \leq \frac{1}{4} \epsilon\left|v_{n}^{2}\right|_{L^{4}}^{4}+\frac{1}{4 \epsilon}\left|v_{n}^{1}\right|_{H^{1}}^{2}
\end{aligned}
$$

where $\epsilon>0$. For the second term on the right hand side of (88), we have

$$
\left|\left\langle v_{n}^{1} \partial_{1} W_{A, 2}^{n}, v_{n}^{2}\right\rangle\right| \leq \varepsilon\left\|v^{n}\right\|_{H^{1}}^{2}+C\left\|v^{n}\right\|_{H}^{2}
$$

Analogously, for the third term on the right hand side of (88), we see that

$$
\left|\left\langle W_{A, 1}^{n} \partial_{1} v_{n}^{2}, v_{n}^{2}\right\rangle\right| \leq C\left\|v^{n}\right\|_{H}^{2}+\varepsilon\left\|v^{n}\right\|_{H^{1}}^{2}
$$

For the last term, by (75) and (76), we have

$$
\left|\left\langle W_{A, 1}^{n} \partial_{x} W_{A, 2}^{n}, v_{n}^{2}\right\rangle\right| \leq C+\varepsilon\left\|v^{n}\right\|_{H^{1}}^{2} .
$$

By (88)-(92), we get

$$
\begin{aligned}
I_{3} \leq & \frac{1}{4 \epsilon}\left\|v_{n}^{2}\right\|_{L^{4}}^{4}+\frac{\epsilon}{4}\left\|v_{n}^{1}\right\|_{H^{1}}^{2} \\
& +3 \varepsilon\left\|v_{n}\right\|_{H^{1}}^{2}+C\left\|v_{n}\right\|_{H}^{2}+C .
\end{aligned}
$$

Analogously, for $I_{2}$, it follows that

$$
\begin{aligned}
I_{2} \leq & \frac{1}{4 \epsilon}\left\|v_{n}^{1}\right\|_{L^{4}}^{4}+\frac{\epsilon}{4}\left\|v_{n}^{2}\right\|_{H^{1}}^{2} \\
& +3 \varepsilon\left\|v_{n}\right\|_{H^{1}}^{2}+C\left\|v_{n}\right\|_{H}^{2}+C .
\end{aligned}
$$

By (80) and the estimates of $I_{1}, I_{2}, I_{3}$, and $I_{4}$, see (86), (87), (93), and (94), we have

$$
\begin{aligned}
\left\langleB \left( v_{n}\right.\right. & \left.\left.+W_{A}^{n}, v_{n}+W_{A}^{n}\right), v_{n}\right\rangle \\
\leq & C\left(1+\left\|v_{n}\right\|_{H}^{2}\right)+\left(\frac{\epsilon}{4}+14 \varepsilon\right)\left\|v_{n}\right\|_{H^{1}}^{2} \\
& +\frac{1}{4 \epsilon}\left\|v_{n}\right\|_{L^{4}}^{4} .
\end{aligned}
$$


For the last term on the left hand side of (79), we have

$$
\begin{aligned}
\left\langlef \left( v_{v}+\right.\right. & \left.\left.W_{A}^{n}\right), v_{n}\right\rangle \\
= & \vartheta\left\|v_{n}\right\|_{L^{4}}^{4}+3 \vartheta \int_{D}\left|v_{n}\right|^{2}\left(v_{n}^{1} W_{A, 1}^{n}+v_{n}^{2} W_{A, 2}^{n}\right) d x \\
& +\vartheta \int_{D}\left|v_{n}\right|^{2}\left|W_{A}^{n}\right|^{2} d x \\
& +\vartheta \int_{D}\left(v_{n}^{1} W_{A, 1}^{n}+v_{n}^{2} W_{A, 2}^{n}\right)\left|W_{A}^{n}\right|^{2} d x \\
& +2 \vartheta \int_{D}\left(\left|W_{A, 1}^{n}\right|^{2}\left|v_{n}^{1}\right|^{2}+\left|W_{A, 2}^{n}\right|^{2}\left|v_{n}^{2}\right|^{2}\right) d x \\
& +4 \vartheta \int_{D} W_{A, 1}^{n} W_{A, 2}^{n} v_{n}^{1} v_{n}^{2} d x \\
\leq & (\vartheta+\varepsilon)\left\|v_{n}\right\|_{L^{4}}^{4}+C\left(1+\left\|v_{n}\right\|_{H}^{2}\right) .
\end{aligned}
$$

By (79), (95), and (96), we get

$$
\begin{aligned}
\frac{1}{2} \frac{\partial}{\partial t}\left\|v_{n}\right\|_{H}^{2}+\left\|v_{n}\right\|_{H^{1}}^{2}+\vartheta\left\|v_{n}\right\|_{L^{4}}^{4} \\
\leq C\left(1+\left\|v_{n}\right\|_{H}^{2}\right)+\left(\frac{\epsilon}{4}+14 \varepsilon\right)\left\|v_{n}\right\|_{H^{1}}^{2} \\
+\left(\frac{1}{4 \epsilon}+\varepsilon\right)\left\|v_{n}\right\|_{L^{4}}^{4} .
\end{aligned}
$$

Rearranging the above inequality, we deduce that

$$
\begin{aligned}
& \frac{1}{2} \frac{\partial}{\partial t}\left\|v_{n}\right\|_{H}^{2}+\left(1-\frac{\epsilon}{4}-14 \varepsilon\right)\left\|v_{n}\right\|_{H^{1}}^{2} \\
& \quad+\left(\vartheta-\frac{1}{4 \epsilon}-\varepsilon\right)\left\|v_{n}\right\|_{L^{4}}^{4} \leq C\left(1+\left\|v_{n}\right\|_{H}^{2}\right) .
\end{aligned}
$$

Let $\epsilon \in(1 / 4 \vartheta, 4)$, and $\varepsilon$ be small enough, such that

$$
1-\frac{\epsilon}{4}-14 \varepsilon>0, \quad \vartheta-\frac{1}{4 \epsilon}-\varepsilon>0 .
$$

So, we integrate with respect to $t$ on both sides of (98) to obtain

$$
\begin{aligned}
& \left\|v_{n}(t)\right\|_{H}^{2}+C_{\epsilon} \int_{0}^{t}\left\|v_{n}(s)\right\|_{H^{1}}^{2} d s \\
& \quad \leq\left\|v_{n}(0)\right\|_{H}^{2}+C t+C \int_{0}^{t}\left\|v_{n}(s)\right\|_{H}^{2} d s,
\end{aligned}
$$

where $C_{\epsilon}=2(1-\epsilon / 4-14 \varepsilon)$, by Gronwall's inequality, we arrive at

$$
\left\|v_{n}(t)\right\|_{H}^{2} \leq\left(\left\|v_{n}(0)\right\|_{H}^{2}+C t\right) e^{C t} \leq C_{T}
$$

By (100) and (101), we have

$$
\int_{0}^{t}\left\|v_{n}(s)\right\|_{H^{1}}^{2} d s \leq C_{T} .
$$

Multiplying $A v_{n}$ on both sides of (78), and integrating with respect to $x \in D$, we have

$$
\begin{gathered}
\left\langle\frac{\partial v_{n}}{\partial t}, A v_{n}\right\rangle+\left\langle A v_{n}, A v_{n}\right\rangle+\left\langle f\left(v_{n}+W_{A}^{n}\right), A v_{n}\right\rangle \\
=\left\langle B\left(v_{n}+W_{A}^{n}, v_{n}+W_{A}^{n}\right), A v_{n}\right\rangle,
\end{gathered}
$$

which is equivalent to

$$
\begin{aligned}
\frac{1}{2} \frac{\partial}{\partial t} \| & v_{n}\left\|_{H^{1}}^{2}+\right\| v_{n} \|_{H^{2}}^{2} \\
=- & \left\langle f\left(v_{n}+W_{A}^{n}\right), A v_{n}\right\rangle \\
& +\left\langle B\left(v_{n}+W_{A}^{n}, v_{n}+W_{A}^{n}\right), A v_{n}\right\rangle .
\end{aligned}
$$

We first estimate the second term on the right hand side of (104) as follows:

$$
\begin{aligned}
&\left\langle B\left(v_{n}+W_{A}^{n}, v_{n}+W_{A}^{n}\right), A v_{n}\right\rangle \\
&=\left\langle v_{n}^{1}+W_{A, 1}^{n} \partial_{1}\left(v_{n}^{1}+W_{A, 1}^{n}\right), A v_{n}^{1}\right\rangle \\
&+\left\langle v_{n}^{2}+W_{A, 2}^{n} \partial_{2}\left(v_{n}^{1}+W_{A, 1}^{n}\right), A v_{n}^{1}\right\rangle \\
&+\left\langle v_{n}^{1}+W_{A, 1}^{n} \partial_{1}\left(v_{n}^{2}+W_{A, 2}^{n}\right), A v_{n}^{2}\right\rangle \\
&+\left\langle v_{n}^{2}+W_{A, 2}^{n} \partial_{2}\left(v_{n}^{2}+W_{A, 2}^{n}\right), A v_{n}^{2}\right\rangle \\
&= J_{1}+J_{2}+J_{3}+J_{4} .
\end{aligned}
$$

For $J_{1}$, we have

$$
\begin{aligned}
J_{1}= & \left\langle v_{n}^{1} \partial_{1} v_{n}^{1}, A v_{n}^{1}\right\rangle+\left\langle v_{n}^{1} \partial_{1} W_{A, 1}^{n}, A v_{n}^{1}\right\rangle \\
& +\left\langle W_{A, 1}^{n} \partial_{1} v_{n}^{1}, A v_{n}^{1}\right\rangle+\left\langle W_{A, 1}^{n} \partial_{1} W_{A, 1}^{n}, A v_{n}^{1}\right\rangle \\
= & k_{1}+k_{2}+k_{3}+k_{4} .
\end{aligned}
$$

For $k_{1}$, we have

$$
k_{1} \leq \varepsilon\left|v_{n}^{1}\right|_{H^{2}}^{2}+C\left|v_{n}^{1}\right|_{L^{4}}^{2} \cdot\left|v_{n}^{1}\right|_{W^{1,4}}^{2}
$$

By interpolation inequality, there exists some $C>0$, such that

$$
\begin{gathered}
\left|v_{n}^{1}\right|_{L^{4}} \leq C\left|v_{n}^{1}\right|_{H}^{1 / 2}\left|v_{n}^{1}\right|_{H^{1}}^{1 / 2}, \\
\left|v_{n}^{1}\right|_{W^{1,4}} \leq C\left|v_{n}^{1}\right|_{H}^{1 / 4}\left|v_{n}^{1}\right|_{H^{2}}^{3 / 4}
\end{gathered}
$$

Then,

$$
\begin{aligned}
k_{1} & \leq \varepsilon\left|v_{n}^{1}\right|_{H^{2}}^{2}+C\left|v_{n}^{1}\right|_{H}^{3 / 2} \cdot\left|v_{n}^{1}\right|_{H^{1}} \cdot\left|v_{n}^{1}\right|_{H^{2}}^{3 / 2} \\
& \leq \varepsilon\left|v_{n}^{1}\right|_{H^{2}}^{2}+\varepsilon\left|v_{n}^{1}\right|_{H^{2}}^{2}+C\left|v_{n}^{1}\right|_{H}^{6}\left|v_{n}^{1}\right|_{H^{1}}^{4} \\
& \leq 2 \varepsilon\left|v_{n}^{1}\right|_{H^{2}}^{2}+C_{T}\left|v_{n}^{1}\right|_{H^{1}}^{4}
\end{aligned}
$$


where the last inequality follows from (101). For $k_{2}$, we deduce that

$$
\begin{aligned}
k_{2} & \leq \varepsilon\left|v_{n}^{1}\right|_{H^{2}}^{2}+C \int_{D}\left(v_{n}^{1}\right)^{2}\left(\partial_{1} W_{A, 1}^{n}\right)^{2} d x \\
& \leq \varepsilon\left|v_{n}^{1}\right|_{H^{2}}^{2}+C\left|v_{n}^{1}\right|_{H}^{2} \\
& \leq \varepsilon\left|v_{n}^{1}\right|_{H^{2}}^{2}+C_{T} .
\end{aligned}
$$

For $k_{3}$, we arrive at

$$
k_{3} \leq C \int_{D}\left|\partial_{1} v_{n}^{1} \cdot A v_{n}^{1}\right| d x \leq \varepsilon\left|v_{n}^{1}\right|_{H^{2}}^{2}+C\left|v_{n}^{1}\right|_{H^{1}}^{2} .
$$

For $k_{4}$, we obtain

$$
k_{4} \leq C+\varepsilon\left|v_{n}^{1}\right|_{H^{2}}^{2} .
$$

By (106) and (109)-(112),

$$
J_{1} \leq 5 \varepsilon\left|v_{n}^{1}\right|_{H^{2}}^{2}+C_{T}\left|v_{n}^{1}\right|_{H^{1}}^{4}+C\left|v_{n}^{1}\right|_{H^{1}}^{2}+C_{T}
$$

Similarly, for $J_{4}$, we infer that

$$
J_{4} \leq 5 \varepsilon\left|v_{n}^{2}\right|_{H^{2}}^{2}+C_{T}\left|v_{n}^{2}\right|_{H^{1}}^{4}+C\left|v_{n}^{2}\right|_{H^{1}}^{2}+C_{T} .
$$

For $J_{2}$, we have

$$
\begin{aligned}
J_{2}= & \left\langle v_{n}^{2} \partial_{2} v_{n}^{1}, A v_{n}^{1}\right\rangle+\left\langle W_{A, 2}^{n} \partial_{2} v_{n}^{1}, A v_{n}^{1}\right\rangle \\
& +\left\langle v_{n}^{2} \partial_{2} W_{A, 1}^{n}, A v_{n}^{1}\right\rangle+\left\langle W_{A, 2}^{n} \partial_{2} W_{A, 1}^{n}, A v_{n}^{1}\right\rangle \\
= & l_{1}+l_{2}+l_{3}+l_{4} .
\end{aligned}
$$

By interpolation inequality and (101), we deduce that

$$
\begin{aligned}
l_{1} & \leq \varepsilon\left|v_{n}^{1}\right|_{H^{2}}^{2}+C\left|v_{n}^{2}\right|_{L^{4}}^{2} \cdot\left|v_{n}^{1}\right|_{W^{1,4}}^{2} \\
& \leq \varepsilon\left|v_{n}^{1}\right|_{H^{2}}^{2}+C\left|v_{n}^{2}\right|_{H} \cdot\left|v_{n}^{2}\right|_{H^{1}} \cdot\left|v_{n}^{1}\right|_{H}^{1 / 2} \cdot\left|v_{n}^{1}\right|_{H^{2}}^{3 / 2} \\
& \leq 2 \varepsilon\left|v_{n}^{1}\right|_{H^{2}}^{2}+C_{T}\left|v_{n}^{2}\right|_{H^{1}}^{4} .
\end{aligned}
$$

For $l_{2}$, we have

$$
l_{2} \leq C \int_{D}\left|\partial_{2} v_{n}^{1}\right| \cdot\left|A v_{n}^{1}\right| d x \leq \varepsilon\left|v_{n}^{1}\right|_{H^{2}}^{2}+C\left|v_{n}^{1}\right|_{H^{1}}^{2}
$$

Similarly, for $l_{3}$,

$$
l_{3} \leq C \int_{D}\left|v_{n}^{2}\right| \cdot\left|A v_{n}^{1}\right| d x \leq \varepsilon\left|v_{n}^{1}\right|_{H^{2}}^{2}+C_{T} .
$$

As for $l_{4}$, we get

$$
l_{4} \leq \varepsilon\left|v_{n}^{1}\right|_{H^{2}}^{2}+C_{T}
$$

By (115)-(119), we arrive at

$$
J_{2} \leq 5 \varepsilon\left\|v_{n}^{1}\right\|_{H^{2}}^{2}+C_{T}\left\|v_{n}^{2}\right\|_{H^{1}}^{4}+C\left\|v_{n}^{1}\right\|_{H^{1}}^{2}+C_{T} .
$$

Analogously to $J_{2}$, we have

$$
J_{3} \leq 5 \varepsilon\left\|v_{n}^{2}\right\|_{H^{2}}^{2}+C_{T}\left\|v_{n}^{1}\right\|_{H^{1}}^{4}+C\left\|v_{n}^{2}\right\|_{H^{1}}^{2}+C_{T} .
$$

By (105) and the estimates of $J_{1}-J_{4}$, see (113), (114), (120), and (121), we get that

$$
\begin{gathered}
\left\langle B\left(v_{n}+W_{A}^{n}, v_{n}+W_{A}^{n}\right), A v_{n}\right\rangle \\
\leq 10 \varepsilon\left\|v_{n}\right\|_{H^{2}}^{2}+C_{T}\left\|v_{n}\right\|_{H^{1}}^{4} \\
+C\left\|v_{n}\right\|_{H^{1}}^{2}+C_{T} .
\end{gathered}
$$

For the first term on the right hand side of (104), we have

$$
\begin{aligned}
& \left|\left\langle f\left(v_{n}+W_{A}^{n}\right), A v_{n}\right\rangle\right| \\
& \quad \leq \varepsilon\left\|v_{n}\right\|_{H^{2}}^{2}+C\left\|v_{n}+W_{A}^{n}\right\|_{L^{6}}^{6} \\
& \quad \leq \varepsilon\left\|v_{n}\right\|_{H^{2}}^{2}+C\left\|v_{n}\right\|_{L^{6}}^{6}+C_{T} \\
& \quad \leq \varepsilon\left\|v_{n}\right\|_{H^{2}}^{2}+C_{T}\left\|v_{n}\right\|_{H^{1}}^{2}\left\|v_{n}\right\|_{H}^{4}+C_{T} \\
& \quad \leq \varepsilon\left\|v_{n}\right\|_{H^{2}}^{2}+C_{T}\left(1+\left\|v_{n}\right\|_{H^{1}}^{2}\right) .
\end{aligned}
$$

By (104), (122), and (123),

$$
\begin{aligned}
& \frac{1}{2} \frac{\partial}{\partial t}\left\|v_{n}\right\|_{H^{1}}^{2}+\left\|v_{n}\right\|_{H^{2}}^{2} \\
& \quad \leq 11 \varepsilon\left\|v_{n}\right\|_{H^{2}}^{2}+C_{T}\left(1+\left\|v_{n}\right\|_{H^{1}}^{2}\right)\left\|v_{n}\right\|_{H^{1}}^{2}+C_{T} .
\end{aligned}
$$

By the Gronwall inequality, we get

$$
\begin{aligned}
\| v_{n}(t) & \|_{H^{1}}^{2} \\
& \leq\left(\left\|v_{n}(0)\right\|_{H^{1}}^{2}+C_{T}\right) e^{C_{T} \int_{0}^{t}\left(1+\left\|v_{n}(s)\right\|_{H^{1}}^{2} d s\right)} \\
& \leq\left(\left\|v_{n}(0)\right\|_{H^{1}}^{2}+C_{T}\right) e^{C_{T}} .
\end{aligned}
$$

Let $n \rightarrow \infty$, by Fatou Lemma,

$$
\|v(t)\|_{H^{1}}^{2} \leq\left(\|v(0)\|_{H^{1}}^{2}+C_{T}\right) e^{C_{T}} .
$$

\section{Invariant Measures}

5.1. Existence. In this section, we will establish the existence of invariant measure for (2). Analogously to [24], we extend the Wiener process $W(t)$ to $\mathbb{R}$ by setting

$$
W(t):=W^{1}(t), \quad t \leq 0,
$$

where $W^{1}(t)$ is another $H$-valued Wiener process satisfying conditions in Lemma 2 and being independent of $W(t)$. For any $\tau \geq 0$, we consider the following equation:

$$
\begin{array}{r}
d u_{\tau}+\left[A u_{\tau}+B\left(u_{\tau}, u_{\tau}\right)+f\left(u_{\tau}\right)\right] d t=d W \\
\text { on }[0, T] \times D, u_{\tau}(-\tau)=0 .
\end{array}
$$

By Theorem 7, we know that there exists unique solution. In order to obtain the invariant measure, we should show that the family of laws $\left\{\mathscr{L}\left(u_{\tau}(0)\right)\right\}_{\tau \geq 0}$ is tight. Since $H^{1+\delta} \subset H^{1}$ 
is compact, for any $\delta>0$, we only need to show that $\left\{\mathscr{L}\left(u_{\tau}(0)\right)\right\}_{\tau \geq 0}$ is bounded in probability in $H^{1+\delta}$. As we know,

$$
W_{A}(t)=\int_{-\infty}^{t} e^{-(t-s) A} d W(s), \quad t \in \mathbb{R}
$$

is the mild solution of (8) with the following initial condition:

$$
W_{A}(0)=\int_{-\infty}^{0} e^{s A} d W(s)
$$

Making the classical change of variable $v_{\tau}(t)=u_{\tau}(t)-W_{A}(t)$, (128) is equivalent to

$$
\begin{aligned}
\frac{d v_{\tau}(t)}{d t}= & A v_{\tau}(t)+B\left(v_{\tau}(t)+W_{A}(t), v_{\tau}(t)+W_{A}(t)\right) \\
& +f\left(v_{\tau}(t)+W_{A}(t)\right),
\end{aligned}
$$

with initial condition

$$
v_{\tau}(-\tau)=-W_{A}(-\tau) .
$$

In order to get the invariant measure of (131), it is enough to show that $v_{\tau}(0)$ is bounded in probability in $H^{1+\delta}$, for some $\delta>0$. That is what we have to do in Theorem 8 below.

Theorem 8. With conditions in Lemma 2, when $9>1 / 4$, there exists an invariant measure for (2).

Proof. Multiplying (131) by $v_{\tau}$ and integrating on $D$, we get

$$
\begin{aligned}
\frac{1}{2} \frac{d}{d t}\left\|v_{\tau}(t)\right\|_{H}^{2}+\left\|v_{\tau}(t)\right\|_{H^{1}}^{2} \\
+\left\langle f\left(v_{\tau}(t)+W_{A}(t)\right), v_{\tau}(t)\right\rangle \\
=\left\langleB \left( v_{\tau}(t)+W_{A}(t), v_{\tau}(t)\right.\right. \\
\left.\left.+W_{A}(t)\right), v_{\tau}(t)\right\rangle .
\end{aligned}
$$

For the third term on the left hand side of (133), we deduce that

$$
\begin{aligned}
\langle f & \left.\left(v_{\tau}(t)+W_{A}(t)\right), v_{\tau}(t)\right\rangle \\
= & \vartheta\left\langle\left|v_{\tau}(t)+W_{A}(t)\right|^{2}\left(v_{\tau}(t)+W_{A}(t)\right), v_{\tau}(t)+W_{A}(t)\right\rangle \\
& -\vartheta\left\langle\left|v_{\tau}(t)+W_{A}(t)\right|^{2}\left(v_{\tau}(t)+W_{A}(t)\right), W_{A}(t)\right\rangle \\
= & \vartheta\left\|v_{\tau}(t)+W_{A}(t)\right\|_{L^{4}}^{4} \\
& -\vartheta\left\langle\left\|v_{\tau}(t)+W_{A}(t)\right\|^{2}\left(v_{\tau}(t)+W_{A}(t)\right), W_{A}(t)\right\rangle \\
\geq & \vartheta \\
& \left.-\vartheta v_{\tau}(t)\left\|_{L^{4}}-\right\| W_{A}(t) \|_{L^{4}}\right]^{4} \\
& -\vartheta\left\langle\left\|v_{\tau}(t)+W_{A}(t)\right\|^{2}\left(v_{\tau}(t)+W_{A}(t)\right), W_{A}(t)\right\rangle \\
\geq & \vartheta\left\|v_{\tau}(t)\right\|_{L^{4}}^{4}-4 \vartheta\left\|v_{\tau}(t)\right\|_{L^{4}}^{3}\left\|W_{A}(t)\right\|_{L^{4}} \\
& -4 \vartheta\left\|v_{\tau}(t)\right\|_{L^{4}}^{1}\left\|W_{A}(t)\right\|_{L^{4}}^{3} \\
& -\vartheta\left\langle\left\|v_{\tau}(t)+W_{A}(t)\right\|^{2}\left(v_{\tau}(t)+W_{A}(t)\right), W_{A}(t)\right\rangle .
\end{aligned}
$$

Substituting (134) into (133), we have

$$
\begin{aligned}
\frac{1}{2} \frac{d}{d t}\left\|v_{\tau}(t)\right\|_{H}^{2}+\left\|v_{\tau}(t)\right\|_{H^{1}}^{2}+\vartheta\left\|v_{\tau}(t)\right\|_{L^{4}}^{4} \\
\leq 4 \vartheta\left\|v_{\tau}(t)\right\|_{L^{4}}^{3}\left\|W_{A}(t)\right\|_{L^{4}} \\
\quad+4 \vartheta\left\|v_{\tau}(t)\right\|_{L^{4}}\left\|W_{A}(t)\right\|_{L^{4}}^{3} \\
\quad+\vartheta\left\langle\left\|v_{\tau}(t)+W_{A}(t)\right\|^{2}\left(v_{\tau}(t)+W_{A}(t)\right), W_{A}(t)\right\rangle \\
+\left\langle B\left(v_{\tau}(t)+W_{A}(t), v_{\tau}(t)+W_{A}(t)\right), v_{\tau}(t)\right\rangle .
\end{aligned}
$$

For the third term on the right hand side of (135), we get by the Young inequality that

$$
\begin{gathered}
\vartheta\left\langle\left\|v_{\tau}(t)+W_{A}(t)\right\|^{2}\left(v_{\tau}(t)+W_{A}(t)\right), W_{A}(t)\right\rangle \\
\leq \varepsilon\left\|v_{\tau}(t)\right\|_{L^{4}}^{4}+C\left\|W_{A}(t)\right\|_{L^{4}}^{4} .
\end{gathered}
$$

For the last term on the right hand side of (135),

$$
\begin{aligned}
\left\langleB \left( v_{\tau}(t)\right.\right. & \left.\left.+W_{A}(t), v_{\tau}(t)+W_{A}(t)\right), v_{\tau}(t)\right\rangle \\
= & \left\langle\left(v_{\tau}(t) \cdot \nabla\right) v_{\tau}(t), v_{\tau}(t)\right\rangle \\
& +\left\langle\left(W_{A}(t) \cdot \nabla\right) v_{\tau}(t), v_{\tau}(t)\right\rangle \\
& +\left\langle\left(v_{\tau}(t) \cdot \nabla\right) W_{A}(t), v_{\tau}(t)\right\rangle \\
& +\left\langle\left(W_{A}(t) \cdot \nabla\right) W_{A}(t), v_{\tau}(t)\right\rangle \\
= & r_{1}+r_{2}+r_{3}+r_{4} .
\end{aligned}
$$

Since $v_{\tau}(t)$ is vector field, we denote it by $v_{\tau}(t)=$ $\left(v_{\tau}^{1}(t), v_{\tau}^{2}(t)\right)$, where $v_{\tau}^{i}(t)$ is real valued function, $i=1,2$. For $r_{1}$, we have

$$
\begin{aligned}
r_{1}= & \left\langle v_{\tau}^{1}(t) \partial_{1} v_{\tau}^{1}(t)+v_{\tau}^{2}(t) \partial_{2} v_{\tau}^{1}(t), v_{\tau}^{1}(t)\right\rangle \\
& +\left\langle v_{\tau}^{1}(t) \partial_{1} v_{\tau}^{2}(t)+v_{\tau}^{2}(t) \partial_{2} v_{\tau}^{2}(t), v_{\tau}^{2}(t)\right\rangle \\
= & \left\langle v_{\tau}^{2}(t) \partial_{2} v_{\tau}^{1}(t), v_{\tau}^{1}(t)\right\rangle \\
& +\left\langle v_{\tau}^{1}(t) \partial_{1} v_{\tau}^{2}(t), v_{\tau}^{2}(t)\right\rangle \\
\leq & -\frac{1}{2}\left\langle\partial_{2} v_{\tau}^{2}(t),\left(v_{\tau}^{1}(t)\right)^{2}\right\rangle \\
& -\frac{1}{2}\left\langle\partial_{1} v_{\tau}^{1}(t),\left(v_{\tau}^{2}(t)\right)^{2}\right\rangle \\
\leq & \frac{1}{4}\left|\partial_{1} v_{\tau}^{1}(t)\right|_{H}^{2}+\frac{1}{4}\left|v_{\tau}^{2}(t)\right|_{L^{4}}^{4} \\
& +\frac{1}{4}\left|\partial_{2} v_{\tau}^{2}(t)\right|_{H}^{2}+\frac{1}{4}\left|v_{\tau}^{1}(t)\right|_{L^{4}}^{4} \\
\leq & \frac{1}{4}\left\|v_{\tau}(t)\right\|_{H^{1}}^{2}+\frac{1}{4}\left\|v_{\tau}(t)\right\|_{L^{4}}^{4} .
\end{aligned}
$$


Similarly for $r_{2}$,

$$
\begin{aligned}
r_{2}= & \left\langle W_{A, 1}(t) \partial_{1} v_{\tau}^{1}(t)+W_{A, 2}(t) \partial_{2} v_{\tau}^{1}(t), v_{\tau}^{1}(t)\right\rangle \\
& +\left\langle W_{A, 1}(t) \partial_{1} v_{\tau}^{2}(t)+W_{A, 2}(t) \partial_{2} v_{\tau}^{2}(t), v_{\tau}^{2}(t)\right\rangle \\
= & -\left\langle\partial_{1} W_{A, 1}(t),\left(v_{\tau}^{1}(t)\right)^{2}\right\rangle \\
& -\left\langle\partial_{2} W_{A, 2}(t),\left(v_{\tau}^{1}(t)\right)^{2}\right\rangle \\
& -\left\langle\partial_{1} W_{A, 1}(t),\left(v_{\tau}^{2}(t)\right)^{2}\right\rangle \\
& -\left\langle\partial_{2} W_{A, 2}(t),\left(v_{\tau}^{2}(t)\right)^{2}\right\rangle \\
\leq & \varepsilon\left\|v_{\tau}(t)\right\|_{L^{4}}^{4}+C\left\|W_{A}(t)\right\|_{H^{1}}^{2} .
\end{aligned}
$$

Analogously to $r_{1}$, we deduce that

$$
\begin{aligned}
r_{3}= & \left\langle v_{\tau}^{1}(t) \partial_{1} W_{A, 1}(t)+v_{\tau}^{2}(t) \partial_{2} W_{A, 1}(t), v_{\tau}^{1}(t)\right\rangle \\
& +\left\langle v_{\tau}^{1}(t) \partial_{1} W_{A, 2}(t)+v_{\tau}^{2}(t) \partial_{2} W_{A, 2}(t), v_{\tau}^{2}(t)\right\rangle \\
\leq & \varepsilon\left\|v_{\tau}(t)\right\|_{L^{4}}^{4}+C\left\|W_{A}(t)\right\|_{H^{1}}^{2} .
\end{aligned}
$$

For $r_{4}$, we have

$$
\begin{aligned}
r_{4}= & \left\langle W_{A, 1}(t) \partial_{1} W_{A, 1}(t)+W_{A, 2}(t) \partial_{2} W_{A, 1}(t), v_{\tau}^{1}(t)\right\rangle \\
& +\left\langle W_{A, 1}(t) \partial_{1} W_{A, 2}(t)+W_{A, 2}(t) \partial_{2} W_{A, 2}(t), v_{\tau}^{2}(t)\right\rangle \\
\leq & \varepsilon\left|v_{\tau}^{1}(t)\right|_{H}^{2}+C\left|W_{A, 1}(t) \partial_{1} W_{A, 1}(t)\right|_{H}^{2} \\
& +\varepsilon\left|v_{\tau}^{1}(t)\right|_{H}^{2}+C\left|W_{A, 2}(t) \partial_{2} W_{A, 1}(t)\right|_{H}^{2} \\
& +\varepsilon\left|v_{\tau}^{2}(t)\right|_{H}^{2}+C\left|W_{A, 1}(t) \partial_{1} W_{A, 2}(t)\right|_{H}^{2} \\
& +\varepsilon\left|v_{\tau}^{2}(t)\right|_{H}^{2}+C\left|W_{A, 2}(t) \partial_{2} W_{A, 2}(t)\right|_{H}^{2} \\
\leq & \varepsilon\left|v_{\tau}^{1}(t)\right|_{H}^{2}+\left|W_{A, 1}(t)\right|_{L^{4}}^{2} \cdot\left|W_{A, 1}(t)\right|_{W^{1,4}}^{2} \\
& +C\left|W_{A, 2}(t)\right|_{L^{4}}^{2} \cdot\left|W_{A, 1}(t)\right|_{W^{1,4}}^{2} \\
& +\varepsilon\left|v_{\tau}^{2}(t)\right|_{H}^{2}+C\left|W_{A, 1}(t)\right|_{L^{4}}^{2} \cdot\left|W_{A, 2}(t)\right|_{W^{1,4}}^{2} \\
& +C\left|W_{A, 2}(t)\right|_{L^{4}}^{2} \cdot\left|W_{A, 2}(t)\right|_{W^{1,4}}^{2} \\
\leq & \varepsilon\left\|v_{\tau}(t)\right\|_{H}^{2}+C\left\|W_{A}(t)\right\|_{L^{4}}^{2} \cdot\left\|W_{A}(t)\right\|_{W^{1,4}}^{2} \cdot
\end{aligned}
$$

Since $\left\{A^{1 / 2} W_{A}(t)\right\}_{t \in \mathbb{R}}$ is a Gaussian process, we infer that

$$
E\left(\left|A^{1 / 2} W_{A}(t)\right|^{4}\right) \leq C\left[E\left(\left|A^{1 / 2} W_{A}(t)\right|^{2}\right)\right]^{2}
$$

Then, with the proof of Lemma 2, we know that $\left\|W_{A}(t)\right\|_{W^{1,4}}^{2}$ is continuous with respect to $t$. By (137)-(141), we have

$$
\begin{aligned}
\left\langle B\left(v_{\tau}(t)+W_{A}(t), v_{\tau}(t)+W_{A}(t)\right), v_{\tau}(t)\right\rangle \\
\leq\left(\frac{1}{4}+\varepsilon\right)\left\|v_{\tau}(t)\right\|_{H^{1}}^{2}+\left(\frac{1}{4}+2 \varepsilon\right)\left\|v_{\tau}(t)\right\|_{L^{4}}^{4} \\
\quad+C\left(\left\|W_{A}(t)\right\|_{H^{1}}^{2}+\left\|W_{A}(t)\right\|_{L^{4}}^{2}\left\|W_{A}(t)\right\|_{W^{1,4}}^{2}\right) .
\end{aligned}
$$

By (135), (136), and (143), we arrive at

$$
\begin{aligned}
\frac{1}{2} \frac{d}{d t}\left\|v_{\tau}(t)\right\|_{H}^{2}+\left\|v_{\tau}(t)\right\|_{H^{1}}^{2}+\vartheta\left\|v_{\tau}(t)\right\|_{L^{4}}^{4} \\
\leq\left(\frac{1}{4}+3 \varepsilon\right)\left\|v_{\tau}(t)\right\|_{L^{4}}^{4}+\left(\frac{1}{4}+\varepsilon\right)\left\|v_{\tau}(t)\right\|_{H^{1}}^{2} \\
+C\left\|W_{A}(t)\right\|_{W^{1,4}}^{4}+C .
\end{aligned}
$$

It is equivalent to

$$
\begin{gathered}
\frac{1}{2} \frac{d}{d t}\left\|v_{\tau}(t)\right\|_{H}^{2}+\left(\frac{3}{4}-\varepsilon\right)\left\|v_{\tau}(t)\right\|_{H^{1}}^{2} \\
+\left(\vartheta-\frac{1}{4}-3 \varepsilon\right)\left\|v_{\tau}(t)\right\|_{L^{4}}^{4} \\
\leq C\left(1+\left\|W_{A}(t)\right\|_{W^{1,4}}^{4}\right) .
\end{gathered}
$$

Since $\vartheta>1 / 4$, let $\varepsilon$ be small enough, such that

$$
\frac{3}{4}-\varepsilon>0 ; \quad \vartheta-\frac{1}{4}-3 \varepsilon>0 .
$$

Then, the above estimates can be changed into

$$
\begin{gathered}
\frac{d}{d t}\left\|v_{\tau}(t)\right\|_{H}^{2}+\alpha_{1}\left\|v_{\tau}(t)\right\|_{H^{1}}^{2}+C_{\nu}\left\|v_{\tau}(t)\right\|_{L^{4}}^{4} \\
\leq C\left(1+\left\|W_{A}(t)\right\|_{W^{1,4}}^{4}\right) .
\end{gathered}
$$

By the Gronwall inequality, we get

$$
\begin{aligned}
\left\|v_{\tau}(t)\right\|_{H}^{2} \leq & \left\|W_{A}(-\tau)\right\|_{H}^{2} e^{-\alpha_{1}(\tau+t)} \\
& +C \int_{-\tau}^{t}\left(1+\left\|W_{A}(s)\right\|_{W^{1,4}}^{4}\right) e^{\alpha_{1}(s-t)} d s \\
\leq & \left\|W_{A}(-\tau)\right\|_{H}^{2} e^{-\alpha_{1}(\tau+t)} \\
& +C \int_{-\infty}^{0}\left(1+\left\|W_{A}(s)\right\|_{W^{1,4}}^{4}\right) e^{\alpha_{1}(s-t)} d s .
\end{aligned}
$$

Similarly to the argument of [26], we will prove that $\left\|W_{A}(t)\right\|_{W^{1,4}}$ has at most polynomial growth, when $t \rightarrow-\infty$ a.s. So, we conclude that

$$
\sup _{0 \leq \tau ; t \leq T}\left\|v_{\tau}(t)\right\|_{H}^{2}<\infty . \quad \text { a.s. }
$$


Multiplying $e^{\delta t}$ on both sides of (147) and integrating with respect to $t$, we have

$$
\begin{aligned}
\int_{-\tau}^{t} e^{\delta s}\left\|v_{\tau}(s)\right\|_{H^{1}}^{2} d s \\
\leq e^{-\delta \tau}\left\|W_{A}(-\tau)\right\|_{H}^{2}+\alpha_{1} \int_{-\tau}^{t} e^{\delta s}\left\|v_{\tau}(s)\right\|_{H}^{2} d s \\
+C \int_{-\tau}^{t}\left(1+\left\|W_{A}(t)\right\|_{W^{1,4}}^{4}\right) e^{\delta s} d s \\
\leq e^{-\delta \tau}\left\|W_{A}(-\tau)\right\|_{H}^{2} \\
\quad+\alpha \int_{-\infty}^{0} e^{\delta s}\left\|v_{\tau}(s)\right\|_{H}^{2} d s \\
\quad+C \int_{-\infty}^{0}\left(1+\left\|W_{A}(t)\right\|_{W^{1,4}}^{4}\right) e^{\delta s} d s .
\end{aligned}
$$

As

$$
\int_{-\infty}^{0}\left(1+\left\|W_{A}(t)\right\|_{W^{1,4}}^{4}\right) e^{\delta s} d s<\infty,
$$

by (149), we have

$$
\sup _{0 \leq \tau ; t \leq T} \int_{-\tau}^{t} e^{\delta s}\left\|v_{\tau}(s)\right\|_{H^{1}}^{2} d s<\infty . \quad \text { a.s. }
$$

By Theorem 7, we know that for problem (131) there exists unique mild solution, which has the following:

$$
\begin{aligned}
v_{\tau}(0)= & e^{\tau A} W_{A}(-\tau) \\
& +\int_{-\tau}^{0} e^{t A} B\left(\left(v_{\tau}(t)+W_{A}(t), v_{\tau}(t)+W_{A}(t)\right)\right) d t \\
& +\int_{-\tau}^{0} e^{t A} f\left(v_{\tau}(t)+W_{A}(t)\right) d t
\end{aligned}
$$

Then, for any $\zeta \in(0, \theta) \cap(0,1 / 4)$, where the $\theta$ is the parameter in Lemma 2,

$$
\begin{aligned}
& \left\|A^{(1+\zeta) / 2} v_{\tau}(0)\right\|_{H} \\
& \leq\left\|e^{\tau A} A^{(1+\zeta) / 2} W_{A}(-\lambda)\right\|_{H} \\
& \quad+\int_{-\tau}^{0} \| A^{(1+\zeta) / 2} e^{t A} B\left(\left(v_{\tau}(t)+W_{A}(t), v_{\tau}(t)+W_{A}(t)\right) \|_{H} d t\right. \\
& \quad+\int_{-\tau}^{0}\left\|A^{(1+\zeta) / 2} e^{t A} f\left(v_{\tau}(t)+W_{A}(t)\right)\right\|_{H} d t .
\end{aligned}
$$

Since

$$
\begin{aligned}
B( & \left.\left(v_{\tau}(t)+W_{A}(t), v_{\tau}(t)+W_{A}(t)\right)\right) \\
= & \left(v_{\tau}(t) \cdot \nabla\right) v_{\tau}(t)+\left(v_{\tau}(t) \cdot \nabla\right) W_{A}(t) \\
\quad & +\left(W_{A}(t) \cdot \nabla\right) v_{\tau}(t)+\left(W_{A}(t) \cdot \nabla\right) W_{A}(t),
\end{aligned}
$$

then,

$$
\begin{aligned}
\| A^{(1+\zeta) / 2} & e^{t A} B\left(\left(v_{\tau}(t)+W_{A}(t), v_{\tau}(t)+W_{A}(t)\right)\right) \|_{H} \\
\leq & \left\|A^{(1+\zeta) / 2} e^{t A}\left[v_{\tau}(t) \cdot \nabla\right] v_{\tau}(t)\right\|_{H} \\
& +\left\|A^{(1+\zeta) / 2} e^{t A}\left[v_{\tau}(t) \cdot \nabla\right] W_{A}(t)\right\|_{H} \\
& +\left\|A^{(1+\zeta) / 2} e^{t A}\left[W_{A}(t) \cdot \nabla\right] v_{\tau}(t)\right\|_{H} \\
& +\left\|A^{(1+\zeta) / 2} e^{t A}\left[W_{A}(t) \cdot \nabla\right] W_{A}(t)\right\|_{H} \\
= & z_{1}+z_{2}+z_{3}+z_{4} .
\end{aligned}
$$

For $z_{1}$, we have

$$
\begin{aligned}
z_{1} \leq & \left|A^{(1+\zeta) / 2} e^{t A}\left[v_{\tau}^{1}(t) \partial_{1} v_{\tau}^{1}(t)\right]\right|_{H} \\
& +\left|A^{(1+\zeta) / 2} e^{t A}\left[v_{\tau}^{2}(t) \partial_{2} v_{\tau}^{1}(t)\right]\right|_{H} \\
& +\left|A^{(1+\zeta) / 2} e^{t A}\left[v_{\tau}^{1}(t) \partial_{1} v_{\tau}^{2}(t)\right]\right|_{H} \\
& +\left|A^{(1+\zeta) / 2} e^{t A}\left[v_{\tau}^{2}(t) \partial_{2} v_{\tau}^{2}(t)\right]\right|_{H} \\
= & z_{1,1}+z_{1,2}+z_{1,3}+z_{1,4} .
\end{aligned}
$$

In the following, we use Theorem 6.13 in chapter two of [27] to estimate them respectively as follows:

$$
\begin{aligned}
z_{1,1} & =\frac{1}{2}\left|e^{t A} A^{(1+\zeta) / 2} \partial_{1}\left(v_{\tau}^{1}\right)^{2}\right|_{H} \\
& \leq \frac{1}{2}\left|e^{t A} A^{(3+2 \zeta) / 4}\left(v_{\tau}^{1}\right)^{2}\right|_{H^{1 / 2}} \\
& \leq C|t|^{-(3+2 \zeta) / 4} e^{\delta t}\left|\left(v_{\tau}^{1}\right)^{2}\right|_{H^{1 / 2}} \\
& \leq C|t|^{-(3+2 \zeta) / 4} e^{\delta t}\left|2 v_{\tau}^{1}(t) A^{1 / 4} v_{\tau}^{1}(t)+R_{4}\right|_{H},
\end{aligned}
$$

the last inequality follows by Theorem A.8 in [25], where $\delta>0, R_{4}=A^{1 / 4}\left(v_{\tau}^{1}\right)^{2}-2 v_{\tau}^{1} A^{1 / 4} v_{\tau}^{1}$, and $\left|R_{4}\right|_{H} \leq$ $C\left|A^{1 / 8} v_{\tau}^{1}(t)\right|_{L^{4}}^{2} \leq C\left|v_{\tau}^{1}(t)\right|_{H^{1}}^{2}$. So, by Hölder inequality and interpolation inequality, we have

$$
z_{1,1} \leq C|t|^{-(3+2 \zeta) / 4} e^{\delta t}\left\|v_{\tau}(t)\right\|_{H^{1}}^{2} .
$$

For $z_{1,2}$, we have

$$
\begin{aligned}
z_{1,2}= & \left|A^{(1+\zeta) / 2} e^{t A}\left[v_{\tau}^{2}(t) \partial_{2} v_{\tau}^{1}(t)\right]\right|_{H} \\
\leq & \left|A^{(1+\zeta) / 2} e^{t A}\left[A^{1 / 4}\left(v_{\tau}^{2}(t) A^{1 / 4} v_{\tau}^{1}(t)\right)\right]\right|_{H} \\
& +\left|A^{(1+\zeta) / 2} e^{t A}\left[A^{1 / 4} v_{\tau}^{1}(t) A^{1 / 4} v_{\tau}^{2}(t)\right]\right|_{H} \\
& +\left|A^{(1+\zeta) / 2} e^{t A} R_{5}\right|_{H},
\end{aligned}
$$

where

$$
\begin{aligned}
R_{5}= & A^{1 / 4}\left[v_{\tau}^{2}(t) A^{1 / 4} v_{\tau}^{1}(t)\right] \\
& -\left[A^{1 / 4} v_{\tau}^{1}(t), A^{1 / 4} v_{\tau}^{2}(t)\right] \\
& -v_{\tau}^{2}(t) A^{1 / 2} v_{\tau}^{1}(t) .
\end{aligned}
$$


Analogously to estimating $z_{1,1}$, we have

$$
\begin{aligned}
z_{1,2} \leq & C|t|^{-(3+2 \zeta) / 4} e^{\delta t}\left|v_{\tau}^{2}(t)\right|_{L^{4}}\left|v_{\tau}^{1}(t)\right|_{W^{1 / 2,4}} \\
& +C|t|^{-(1+\zeta) / 2} e^{\delta t}\left|v_{\tau}^{1}(t)\right|_{W^{1 / 2,4}}\left|v_{\tau}^{2}(t)\right|_{W^{1 / 2,4}} \\
\leq & C\left(|t|^{-(3+2 \zeta) / 4}+|t|^{-(1+\zeta) / 2}\right) e^{\delta t}\left\|v_{\tau}(t)\right\|_{W^{1 / 2,4}}^{2} \\
\leq & C\left(|t|^{-(3+2 \zeta) / 4}+|t|^{-(1+\zeta) / 2}\right) e^{\delta t}\left\|v_{\tau}(t)\right\|_{H^{1}}^{2}
\end{aligned}
$$

Similarly, we can get the same estimates for $z_{1,3}$ and $z_{1,4}$. Therefore,

$$
z_{1} \leq C\left(|t|^{-(3+2 \zeta) / 4}+|t|^{-(1+\zeta) / 2}\right) e^{\delta t}\left\|v_{\tau}(t)\right\|_{H^{1}}^{2} .
$$

Analogously to estimating $z_{1}$, we can get for $z_{2}, z_{3}$, and $z_{4}$ that

$$
\begin{aligned}
z_{2} \leq & C\left(|t|^{-(3+2 \zeta) / 4}+|t|^{-(1+\zeta) / 2}\right) \\
& \times e^{\delta t}\left(\left\|W_{A}(t)\right\|_{H^{1}}^{2}+\left\|v_{\tau}(t)\right\|_{H^{1}}^{2}\right), \\
z_{3} \leq & C\left(|t|^{-(3+2 \zeta) / 4}+|t|^{-(1+\zeta) / 2}\right) \\
& \times e^{\delta t}\left(\left\|W_{A}(t)\right\|_{H^{1}}^{2}+\left\|v_{\tau}(t)\right\|_{H^{1}}^{2}\right), \\
z_{4} \leq & C\left(|t|^{-(3+2 \zeta) / 4}+|t|^{-(1+\zeta) / 2}\right) \\
& \times e^{\delta t}\left\|W_{A}(t)\right\|_{H^{1}}^{2} .
\end{aligned}
$$

So, by (163)-(164) and (156), we get

$$
\begin{gathered}
\left\|A^{(1+\zeta) / 2} e^{t A} B\left(\left(v_{\tau}(t)+W_{A}(t), v_{\tau}(t)+W_{A}(t)\right)\right)\right\|_{H} \\
\leq C\left(|t|^{-(3+2 \zeta) / 4}+|t|^{-(1+\zeta) / 2}\right) \\
\times e^{\delta t}\left(\left\|W_{A}(t)\right\|_{H^{1}}^{2}+\left\|v_{\tau}(t)\right\|_{H^{1}}^{2}\right) .
\end{gathered}
$$

For the third term on the right hand side of (154), we obtain

$$
\begin{aligned}
\| A^{(1+\zeta) / 2} & e^{t A} f\left(v_{\tau}(t)+W_{A}(t)\right) \|_{H} \\
\leq & C|t|^{-(1+\zeta) / 2} e^{\delta t}\left(\left\|v_{\tau}(t)\right\|_{L^{6}}^{3}+\left\|W_{A}(t)\right\|_{L^{6}}^{3}\right) \\
\leq & C|t|^{-(1+\zeta) / 2} \\
& \times e^{\delta t}\left(\left\|v_{\tau}(t)\right\|_{H} \cdot\left\|v_{\tau}(t)\right\|_{H^{1}}^{2}+\left\|W_{A}(t)\right\|_{H^{1}}^{3}\right) \\
\leq & C|t|^{-(1+\zeta) / 2} e^{\delta t} \\
& \times e^{\delta t}\left(\left\|v_{\tau}(t)\right\|_{H^{1}}^{2}+\left\|W_{A}(t)\right\|_{H^{1}}^{2}\right),
\end{aligned}
$$

since $\left\|v_{\tau}(t)\right\|_{H}$ and $e^{\delta t}\left\|W_{A}(t)\right\|_{H^{1}}^{2}$ are bounded for $t, \tau \in$ $(-\infty, T]$, the last inequality follows. For the first term on the right hand side of (154), we have

$$
\left\|e^{\tau A} A^{(1+\zeta) / 2} W_{A}(-\tau)\right\|_{H} \leq e^{-\delta \tau}\left\|A^{(1+\zeta) / 2} W_{A}(-\tau)\right\|_{H^{*}}
$$

Similar to [26], we can prove that $\left\|A^{(1+\zeta) / 2} W_{A}(-\tau)\right\|_{H}$ has at most polynomial growth when $\tau \rightarrow \infty$. For the reader convenience, we sketch a proof. By Lemma 2, we know that $W(t)-W(s)$ is a $D\left(A^{\theta / 2}\right)$ valued Brownian motion, for $s \leq$ $t \leq 0$. So, by the law of iterated logarithm, we have

$$
w_{n}:=\sup _{n \leq s \leq t \leq n+1} \frac{\|W(t)-W(s)\|_{H^{\theta}}}{|t-s|^{1 / 2+(\zeta-\theta) / 4}}<\infty, \quad \text { a.s. } n \in \mathbb{Z} .
$$

Obviously, $w_{n}$ is a i.i.d sequence. By the law of large numbers, there exists an integer-valued random variable $n_{0}(w)>0$, when $n \geq n_{0}(w)$, we have

$$
\frac{w_{-n}}{n} \leq \frac{w_{-n}+\cdots+w_{-1}}{n} \leq E w_{0}+1<\infty .
$$

This implies that

$$
w_{-n} \leq C_{0}(w) n
$$

for all $n>0$. In other words,

$$
\|W(t)-W(s)\|_{H^{\theta}} \leq C_{0}(w)|[s]| \cdot|t-s|^{1 / 2+(\zeta-\theta) / 4},
$$

when $s \leq t \leq[s]+1$. By the law of iterated logarithm, we have

$$
\|W(t)\|_{H^{\theta}} \leq C_{1}(w)|t|, \quad t \in(-\infty, 0],
$$

for some positive random variable. By Theorem 5.14 in [23], we know that

$$
W_{A}(t)=\int_{-\infty}^{t} A e^{-(t-s) A}(W(t)-W(s)) d s .
$$

So, we have that

$$
\begin{aligned}
\left\|A^{(1+\zeta) / 2} W_{A}(t)\right\|_{H} & \\
\leq & \int_{-\infty}^{t}\left\|A^{1+1 / 2+\zeta / 2} e^{-(t-s) A}(W(t)-W(s))\right\|_{H} d s \\
& =\int_{-\infty}^{t}\left\|A^{1+1 / 2+(\zeta-\theta) / 2} e^{-(t-s) A}\left[A^{\theta / 2}(W(t)-W(s))\right]\right\|_{H} d s \\
\leq & \int_{-\infty}^{t} \frac{e^{-\delta(t-s)}}{|t-s|^{1+1 / 2+(\zeta-\theta) / 2}}\|W(t)-W(s)\|_{H^{\theta}} d s \\
\leq & \int_{[t]-1}^{t} \frac{e^{-\delta(t-s)}}{|t-s|^{1+(\zeta-\theta) / 4}} \cdot \frac{\|W(t)-W(s)\|_{H^{\theta}}}{|t-s|^{1 / 2+(\zeta-\theta) / 4}} \\
& +\int_{-\infty}^{[t]-1} \frac{e^{-\delta(t-s)}}{|t-s|^{1+(\zeta-\theta) / 4}} \cdot \frac{C_{1}(w)(|t|+|s|)}{|t-s|^{1 / 2+(\zeta-\theta) / 4}} \\
\leq & \int_{[t]-1}^{t} \frac{e^{-\delta(t-s)}}{|t-s|^{1+(\zeta-\theta) / 4}} \cdot C_{0}(w)|[s]| d s \\
& +\int_{-\infty}^{[t]-1} e^{-\delta(t-s)} C_{1}(w)(|t|+|s|) \\
\leq & \left(C_{0}(w)+C_{1}(w)\right)(|t|+1),
\end{aligned}
$$

since $s \leq[t]-1$, the fourth inequality follows. By (167) and (174), we know that

$$
\sup _{\tau \geq 0}\left\|e^{\tau A} A^{(1+\zeta) / 2} W_{A}(-\tau)\right\|_{H}<\infty \text {, a.s. }
$$


If we let $\zeta=1 / 2<\theta$, repeating the argument of (174), we can see that $\left\|W_{A}(t)\right\|_{W^{1,4}}$ also has at most polynomial growth, when $t \rightarrow-\infty$ a.s., since we have the Sobolev embedding $H^{3 / 2} \subset W^{1,4}$. Consider the second term on the right hand side of (154), by (165),

$$
\begin{aligned}
\int_{-\tau}^{0}\left\|A^{(1+\zeta) / 2} e^{t A} B\left(\left(v_{\tau}(t)+W_{A}(t), v_{\tau}(t)+W_{A}(t)\right)\right)\right\|_{H} d t \\
\leq \int_{-\tau}^{0} C\left(|t|^{-(3+2 \zeta) / 4}+|t|^{-(1+\zeta) / 2}\right) \\
\quad \times e^{\delta t}\left(\left\|W_{A}(t)\right\|_{H^{1}}^{2}+\left\|v_{\tau}(t)\right\|_{H^{1}}^{2}\right) d t \\
\leq \int_{-1}^{0} C\left(|t|^{-(3+2 \zeta) / 4}+|t|^{-(1+\zeta) / 2}\right) \\
\quad \times e^{\delta t}\left(\left\|W_{A}(t)\right\|_{H^{1}}^{2}+\left\|v_{\tau}(t)\right\|_{H^{1}}^{2}\right) d t \\
\quad+\int_{-\infty}^{-1} C e^{\delta t}\left(\left\|W_{A}(t)\right\|_{H^{1}}^{2}+\left\|v_{\tau}(t)\right\|_{H^{1}}^{2}\right) d t<\infty,
\end{aligned}
$$

where the last inequality follows by (152). Analogously, we can prove that

$$
\begin{aligned}
\int_{-\tau}^{0} & \left\|A^{(1+\zeta) / 2} e^{t A} f\left(v_{\tau}(t)+W_{A}(t)\right)\right\|_{H} d t \\
\leq & \int_{-\tau}^{0} C\left(|t|^{-(1+\zeta) / 2}\right) \\
& \times e^{\delta t}\left(\left\|v_{\tau}(t)\right\|_{H}\left\|v_{\tau}(t)\right\|_{H^{1}}^{2}+\left\|W_{A}(t)\right\|_{H^{1}}^{3}\right) \\
<\infty &
\end{aligned}
$$

where we used (149) and (152) for the last inequality. By (154) and (175)-(177), we get

$$
\left\|A^{(1+\zeta) / 2} v_{\tau}(0)\right\|_{H} \leq \xi(w), \quad \text { a.s., }
$$

for some positive random variable $\xi(w)$. As $H^{1+\delta} \subset H^{1}$ is compact, by Prohorov Theorem, we know that the family of laws for $\left(v_{\tau}(0)\right)_{\tau \geq 0}$ taking values in $H^{1}$ is tight. Since $v_{\tau}(0)=$ $u_{\tau}(0)-W_{A}(0)$, then so does the law of $\left(u_{\tau}(0)\right)_{\tau \geq 0}$ taking values in the same space. For $t \geq 0$, set

$$
\left(P_{t} f\right)(x)=E f(u(t, . ; 0, x)),
$$

where $f \in C_{b}\left(H_{0}^{1}\right)$. Following the arguments in [24], for all $t_{0}<s<t$ and all $u_{t_{0}} \in H_{0}^{1}$, by proving

$$
E\left(f\left(u\left(t ; t_{0}, u_{t_{0}}\right)\right) \mid \mathscr{F}_{s}\right)=P_{t-s}\left(u\left(s ; t_{0}, u_{t_{0}}\right)\right),
$$

we can show that $u$ is a Markov process. Here, $\mathscr{F}_{s}$ is the $\sigma$ algebra generated by $W(r)$ for $r \leq s$. So, $\left(P_{t}\right)_{t \geq 0}$ is the Markov semigroup. Define a dual semigroup $P_{t}^{*}$ in the space $P\left(H_{0}^{1}\right)$ of probability measures on $H_{0}^{1}$ as follows:

$$
\int_{H_{0}^{1}} f d\left(P_{t}^{*} \mu\right)=\int_{H_{0}^{1}} P_{t} f d \mu .
$$

Let $\mu_{\tau}$ be the law of $u_{\tau}(0)$, which is the solution of (2) with initial condition $u(-\tau)=0$. Then, we have

$$
\begin{aligned}
\mu_{\tau}(f) & =E f\left(u_{-\tau}(0)\right)=\operatorname{Ef}(u(\tau, \cdot ; 0,0)) \\
& =\left(P_{\tau} f\right)(0)=\int_{H_{0}^{1}} P_{\tau} f d \delta_{0} \\
& =\int_{H_{0}^{1}} f d\left(P_{\tau}^{*} \delta_{0}\right),
\end{aligned}
$$

where we use the fact that $u(\tau, \cdot ; 0,0)$ and $u_{\tau}(0)$ have the same law, the second equality follows. Therefore,

$$
P_{\tau_{1}}^{*} \mu_{\tau}=\mu_{\tau+\tau_{1}} .
$$

Since $\left(\mu_{\tau}\right)_{\tau \geq 0}$ is tight, then by Prokhorov theorem, we know that $\left(\mu_{\tau}\right)_{\tau>0}$ is relatively compact. We can choose a subsequence of $\left(\mu_{\tau}\right)_{\tau \geq 0}$ denoted by $\left(\mu_{\tau_{n}}\right)_{n \in \mathbb{N}}$ such that for $\mu \in$ $P\left(H^{\sigma}\right)$,

$$
\begin{aligned}
\int_{H_{0}^{1}} & \left(P_{t} f\right)(x) \mu(d x) \\
& =\lim _{n \rightarrow \infty} \int_{H_{0}^{1}}\left(P_{t} f\right)(x) \mu_{\tau_{n}}(d x) \\
& =\lim _{n \rightarrow \infty} \int_{H_{0}^{1}} f(x) P_{t}^{*} \mu_{\tau_{n}}(d x) \\
& =\lim _{n \rightarrow \infty} \int_{H_{0}^{1}} f(x) \mu_{\tau_{n}+t}(d x) \\
& =\int_{H_{0}^{1}} f(x) \mu(d x) .
\end{aligned}
$$

5.2. Uniqueness. The main result of this part is as follows.

Theorem 9. Assume $\theta>1 / 2$ in Lemma 2 and $9>1 / 4$; then,

(i) the stochastic Burgers equation (2) has a unique invariant measure $\mu$;

(ii) for all $u_{0} \in H_{0}^{1} \varphi, H_{0}^{1} \rightarrow \mathbb{R}$, such that $\int_{H_{0}^{1}}|\varphi| d \mu<\infty$,

$$
\lim _{T \rightarrow \infty} \frac{1}{T} \int_{0}^{T} \varphi\left(u\left(t ; u_{0}\right)\right) d t=\int_{H_{0}^{1}} \varphi d \mu \quad \text { a.s.; }
$$

(iii) for every Borel measure $\mu^{*}$ on $H_{0}^{1}$, one has that

$$
\left\|P_{t}^{*} \mu^{*}-\mu\right\|_{T V} \longrightarrow 0 \text { as } t \longrightarrow \infty,
$$

where $\|\cdot\|_{T V}$ stands for the total variation of a measure. In particularly, one has that

$$
P_{t}^{*} \mu^{*}(B) \longrightarrow \mu(B), \quad \text { as } t \longrightarrow \infty,
$$

for every Borel set $B \in \mathscr{B}\left(H_{0}^{1}\right)$ (the Borel $\sigma$-algebra of $\left.H_{0}^{1}\right)$. 
In order to prove Theorem 9, we only need Theorem 10 below, see [28, Theorem 4.2.1]. We define $P(t, x, \cdot), t>0, x \in$ $H_{0}^{1}$, to be the transition probability measure that is,

$$
P(t, x, B)=P_{t}^{*} \delta_{x}(B)=P(u(t ; x) \in B)
$$

for $B \in \mathscr{B}\left(H_{0}^{1}\right)$.

Theorem 10. Assume that the probability measures $P(t$, $x, \cdot), t>0, x \in H_{0}^{1}$, are all equivalent, in the sense that they are mutually absolutely continuous. Then, Theorem 9 holds true.

In the following, we will prove the irreducibility and the strong Feller property in $H_{0}^{1}$ to get the equivalence of the measure $P(t, x, \cdot)$. For the two notations, we outline them below. For $y \in H_{0}^{1}, \varepsilon>0$, let

$$
B(y, \varepsilon)=\left\{x \in H_{0}^{1} ;\|x-y\|_{H^{1}}<\varepsilon\right\} .
$$

(I) For any $x, y \in H_{0}^{1}$, such that for all $\varepsilon>0$,

$$
P(t, x, B(y, \varepsilon))>0
$$

for each $t>0$.

(S) For all $O \in \mathscr{B}\left(H_{0}^{1}\right)$, every $t>0$, and all $x_{n}, x \in H_{0}^{1}$ such that $x_{n} \rightarrow x$ in $H_{0}^{1}$, it holds that

$$
P\left(t, x_{n}, O\right) \longrightarrow P(t, x, O) \text {. }
$$

Before checking the condition (I), we need Lemma 11 below. For $x \in H_{0}^{1}$ and $\phi:[0, T] \rightarrow H_{0}^{1}$, set

$$
u(t, x, \phi)=v(t, x, \phi)+\phi(t)
$$

where $v(t, x, \phi)$ is solution of the following equation:

$$
\frac{d v}{d t}+A v+B(v+\phi, v+\phi)+f(v+\phi)=0
$$

for $t \in[0, T]$, with initial condition $v(0)=x$. As it is proved in previously this equation has a unique solution as follows:

$$
v \in C\left([0, T] ; H_{0}^{1}\right)
$$

when $x \in H_{0}^{1}$ and $\phi \in C\left([0, T] ; H_{0}^{1}\right)$.

Lemma 11. Define $\Psi(\phi)=u(\cdot, x, \phi)$; then,

(i) the mapping

$$
\Psi: C_{0}\left([0, T] ; H^{3 / 2}\right) \longrightarrow C\left([0, T] ; H_{0}^{1}\right)
$$

is continuous, where $C_{0}([0, T] ; B):=\{h \quad \epsilon$ $C([0, T] ; B) ; h(0)=0\}$ for Banach space $B$;

(ii) for every $x, y \in H^{3 / 2}$ and $T>0$ there exists $\bar{z} \epsilon$ $C_{0}\left([0, T] ; H^{3 / 2}\right)$ such that $u(T, x, \bar{z})=y$.
Proof. (i) is proved by (A.30) in the Appendix. To prove (ii), let $x, y, \in H^{3 / 2}$ and $T>0$, define $\bar{u}$ as

$$
\begin{gathered}
\bar{u}(t)=e^{-t A} x, \quad t \in\left[0, t_{0}\right], \\
\bar{u}(t)=e^{-(T-t) A} y, \quad t \in\left[t_{1}, T\right], \\
\bar{u}(t)=\bar{u}\left(t_{0}\right)+\frac{t-t_{0}}{t_{1}-t_{0}}\left(\bar{u}\left(t_{1}\right)-\bar{u}\left(t_{0}\right)\right), \\
t \in\left(t_{0}, t_{1}\right) .
\end{gathered}
$$

Obviously, $\bar{u}(t) \in C\left([0, T] ; H^{3 / 2}\right)$. Define $\bar{v}$ as the solution of the following equation:

$$
\frac{d}{d t} \bar{v}+A \bar{v}+B(\bar{u}, \bar{u})+f(\bar{u})=0
$$

with initial condition $\bar{v}(0)=x$; then $\bar{v} \in C\left([0, T] ; H^{3 / 2}\right)$. Set $\bar{z}=\bar{u}-\bar{v}$; then it satisfies all the requirements of the lemma.

Proposition 12. With conditions in Theorem 9, the irreducibility property (I) is satisfied.

Proof. Let $x \in H^{3 / 2}$ and $\bar{z}$ be the same as (ii) in Lemma 11. By the above lemma, we have that for $\varepsilon>0$, we can find $\delta>0$, such that

$$
\|z-\bar{z}\|_{C_{0}\left([0, T] ; H^{3 / 2}\right)}<\delta
$$

implies that

$$
\|u(\cdot, x, z)-u(\cdot, x, \bar{z})\|_{C\left([0, T] ; H^{1}\right)}<\varepsilon .
$$

If $\theta>1 / 2$ in Lemma 2 , and denote $z$ and $\bar{z}$ the corresponding Ornstein-Uhlenbeck process satisfying conditions in the lemma, then $z, \bar{z} \in C\left([0, T] ; H^{3 / 2}\right)$. Choose $\delta_{1}>0$ such that $\delta_{1}<\delta$ and

$$
z \in U_{\delta_{1}}=:\left\{z \in C_{0}\left([0, T] ; H^{3 / 2}\right) ;\|z-\bar{z}\|_{C\left([0, T] ; H^{3 / 2}\right)}<\delta_{1}\right\} .
$$

Then, for $z \in U_{\delta_{1}}$, we have that

$$
\|u(T,, x, z)-y\|_{H^{1}}<\varepsilon .
$$

Recall now that the solution $u$ of the stochastic Burgers equation is equal to $\Psi(z), z$ being the Ornstein-Uhlenbeck process. Then, it remains to show that

$$
P\left\{z(\cdot, w) \in U_{\delta_{1}}\right\}>0
$$

But this is obviously true. So far, we have proved that for for all $t>0$, for all $x, y \in H^{3 / 2}$, for all $\varepsilon>0$,

$$
P(t, x, B(y, \varepsilon))>0 .
$$


Next, we will prove for all $x_{0} \in H_{0}^{1}, y_{0} \in H^{3 / 2}$, the above inequality also holds. Indeed, for $0<h<t$, by ChapmanKolmogorov equation, we have

$$
\begin{aligned}
P( & \left.t, x_{0}, B\left(y_{0}, \varepsilon\right)\right) \\
& =\int_{H_{0}^{1}} P\left(t-h, x_{0}, d y\right) P\left(h, y, B\left(y_{0}, \varepsilon\right)\right) \\
& =\int_{H^{3 / 2}} P\left(t-h, x_{0}, d y\right) P\left(h, y, B\left(y_{0}, \varepsilon\right)\right)>0 .
\end{aligned}
$$

Since $P\left(t-h, x_{0}, H^{3 / 2}\right)=1$, we will extend (204) to the case for all $x_{0} \in H_{0}^{1}, y_{0} \in H_{0}^{1}$. If this is not true, there exists $t_{0}>$ $0, x_{0}, y_{0} \in H_{0}^{1}, \varepsilon>0$ such that

$$
P\left(t_{0}, x_{0}, B\left(y_{0}, \varepsilon\right)\right)=0 .
$$

Then, we can choose $y_{1} \in H^{3 / 2}, \varepsilon_{1}>0$ such that $B\left(y_{1}, \varepsilon_{1}\right) \subset$ $B\left(y_{0}, \varepsilon\right)$. By (204), we have

$$
P\left(t_{0}, x_{0}, B\left(y_{1}, \varepsilon_{1}\right)\right)>0,
$$

which is contrary to (205).

In this part, it is time to check the condition (S).

We will first obtain the strong Feller property in $H_{0}^{1}$ for modified Burgers equation (208) below, then let $R \rightarrow \infty$ to check the condition $(S)$.

Fix $R>0$, let $K_{R}:\left[0, \infty\left[\rightarrow\left[0, \infty\left[\right.\right.\right.\right.$ satisfy $K_{R} \in C^{1}\left(\mathbb{R}_{+}\right)$ such that $\left|K_{R}\right| \leq 1,\left|K_{R}^{\prime}\right| \leq 2$ and

$$
\begin{gathered}
K_{R}=1, \quad \text { if } x<R, \\
K_{R}=0, \quad \text { if } x \geq R+1 .
\end{gathered}
$$

Consider the following equation:

$$
\begin{aligned}
& d u_{R}(t)+A u_{R}(t) d t \\
& \quad+K_{R}\left(\left\|u_{R}(t)\right\|_{H^{1}}^{2}\right) B\left(u_{R}(t), u_{R}(t)\right) d t \\
& \quad+K_{R}\left(\left\|u_{R}(t)\right\|_{H^{1}}^{2}\right) f\left(u_{R}\right)(t)=d W(t) .
\end{aligned}
$$

Proposition 13. There exists a unique mild solution $u_{R}(\cdot, w) \in$ $C\left([0, T] ; H_{0}^{1}\right)$ for (208) which is Markov process with the Feller property in $H_{0}^{1}$, that is for every $R>0, t>0$, there exists a constant $L=L(t, R)>0$ such that

$$
\left|P_{t}^{(R)} \phi(x)-P_{t}^{(R)} \phi(y)\right| \leq L\|x-y\|_{H^{1}}
$$

holds for all $x, y \in H_{0}^{1}$, and all $\phi \in C_{b}\left(H_{0}^{1}\right) \leq 1$, where $P_{t}^{(R)} \phi(x):=\int_{H_{0}^{1}} \phi(y) P_{R}(t, x, d y), P_{R}(t, x, \cdot)$ is the transition probabilities corresponding to (204).

Proof. The proof of existence and uniqueness is similar to Section 2. Let $\phi_{1}=\phi_{2}$ in (A.28), by the Gronwall inequality, we know that $u_{R}$ is Lipschitz continuous with respect to initial value. Using the method in Proposition 4.3.3 in [24], we can prove that the solution is a Markov process. To prove the Fell property, we first consider the following Galerkin approximations of (208). Let $P_{n}$ be the orthogonal projection in $H$ defined as $P_{n} x=\sum_{j=1}^{n}\left\langle x, e_{j}\right\rangle e_{j}, x \in H$. Clearly, $H_{n}:=$ $P_{n} H$ for every $n$. Consider the equation in $H_{n}$ as follows:

$$
\begin{aligned}
& d u_{n}^{(R)}(t)+A u_{n}^{(R)}(t) d t \\
& \quad+K_{R}\left(\left\|u_{n}^{(R)}(t)\right\|_{H^{1}}^{2}\right) P_{n} B\left(u_{n}^{(R)}(t), u_{n}^{(R)}(t)\right) \\
& \quad+K_{R}\left(\left\|u_{n}^{(R)}(t)\right\|_{H^{1}}^{2}\right) f\left(u_{n}^{(R)}\right)(t)=d W(t),
\end{aligned}
$$

with initial condition $u_{n}^{(R)}(0)=P_{n} u_{0}$. This is a finitedimensional equation with globally Lipschitz nonlinear functions, so it has a unique progressively measurable solution with $P$-a.e. trajectory $u_{n}^{(R)}(\cdot, w) \in C\left([0, T] ; H_{n}\right)$, which is also a Markov process in $H_{n}$ with associated semigroup $P_{n, t}^{(R)}$ defined as

$$
P_{n, t}^{(R)} \phi(x)=E \phi\left(u_{n}^{(R)}(t ; x)\right),
$$

for all $x \in H_{n}$ and $\phi \in C_{b}\left(H_{n}\right)$. For every $R>0, t>0$, we can prove that there exists a constant $L=L(t, R)>0$ such that

$$
\left|P_{n, t}^{(R)} \phi(x)-P_{n, t}^{(R)} \phi(y)\right| \leq L\|x-y\|_{H^{1}}
$$

hold for all $n \in \mathbb{N}, x, y \in H_{n}$, and all $\phi \in C_{b}\left(H_{n}\right)$ with $\|\phi\|_{H^{1}} \leq$ 1. Indeed, the following remarkable formula holds true for the differential in $x$ of $P_{n, t}^{(R)} \phi$ [29]:

$$
\begin{gathered}
D_{x} P_{n, t}^{(R)} \phi(x) \cdot h \\
=\frac{1}{t} E\left(\phi ( u _ { n } ^ { ( R ) } ( t ; x ) ) \int _ { 0 } ^ { t } \left\langle\left(P_{n} Q Q^{*} P_{n}\right)^{-1 / 2} D_{x} u_{n}^{(R)}(s ; x)\right.\right. \\
\left.\left.\cdot h, d \beta_{n}(s)\right\rangle\right),
\end{gathered}
$$

for all $h \in H_{n}$, where $\beta_{n}$ is a $n$-dimensional standard Wiener process with incremental covariance $P_{n} Q$ and $Q$ is the covariance operator of $W(t)$. Obviously, $Q$ is nonnegative, adjoint, Hilbert-Schmidt operator with inverse. Since the eigenvalues $\alpha_{n}$ of the Stokes operator $A$, in 2 -space dimension, behave like $n$, let $\theta=1 / 2+\varepsilon$ for some $\varepsilon>0$, in Lemma 2 , we have $D(A) \subset \mathscr{R}(Q) \subset D\left(A^{3 / 4}\right)$, where $\mathscr{R}(Q)$ is the image of $Q$. Therefore,

$$
\begin{aligned}
& \left|D_{x} P_{n, t}^{(R)} \phi(x) \cdot h\right| \\
& \quad \leq \frac{1}{t} E\left(\int_{0}^{t}\left\|\left(P_{n} Q Q^{*} P_{n}\right)^{-1 / 2} D_{x} u_{n}^{(R)}(s ; x) \cdot h\right\|_{H}^{2} d s\right)^{1 / 2} .
\end{aligned}
$$

Since for $y \in H_{n}$

$$
\begin{aligned}
& \left\|\left(P_{n} Q Q^{*} P_{n}\right)^{-1 / 2} y\right\|_{H}^{2} \\
& =\left\langle\left(P_{n} Q Q^{*} P_{n}\right)^{-1} y, y\right\rangle \\
& =\left\langle\left(A P_{n} Q Q^{*} P_{n} A\right)^{-1} A y, A y\right\rangle \leq C\|y\|_{H^{2}}^{2},
\end{aligned}
$$


it follows that

$$
\begin{aligned}
& \left|D_{x} P_{n, t}^{(R)} \phi(x) \cdot h\right| \\
& \quad \leq \frac{1}{t} C E\left(\int_{0}^{t}\left\|D_{x} u_{n}^{(R)}(s ; x) \cdot h\right\|_{H^{2}}^{2} d s\right)^{1 / 2} \\
& \quad \leq \frac{1}{t} C(R)\|h\|_{H^{1}},
\end{aligned}
$$

where the last inequality follows by the Estimate 4 of the Appendix (note that $C(R)$ is independent of $x \in H_{n}$ and $n \in \mathbb{N})$. Indeed, $u_{n}^{(R)}(t, x)$ is given by $v_{n}(t, x)+P_{n} z(t)$, where $z$ is the Ornstein-Uhlenbeck process, and $v_{n}$ is the solution of (A.2). Therefore,

$$
\begin{aligned}
& \left|P_{n, t}^{(R)} \phi(x)-P_{n, t}^{(R)} \phi(y)\right| \\
& \quad \leq \sup _{\|h\|_{H^{1}} \leq 1, k \in H_{n}}\left|D_{x} P_{n, t}^{(R)} \phi(k) \cdot h\right| \cdot\|x-y\|_{H^{1}} \\
& \quad \leq \frac{1}{t} C(R)\|x-y\|_{H^{1}} .
\end{aligned}
$$

In the following step, we will let $n \rightarrow \infty$ to get the Fell property for (208). Let $x \in H_{0}^{1}$ and $\phi \in C_{b}\left(H_{0}^{1}\right)$ be given. From the Appendix, Remark A.1, we know that $u_{n}^{(R)}(t)$ converges to $u^{(R)}(t)$ strongly in $L^{2}\left(0, T ; H_{0}^{1}\right), p$-a.s.. By the boundedness and continuous of $\phi$ as well as Lebesgue dominated convergence theorem, we have

$$
E \int_{0}^{T}\left|\phi\left(u_{n}^{(R)}(, ; x)\right)-\phi\left(u^{(R)}(, ; x)\right)\right| d t \longrightarrow 0,
$$

which implies that for some subsequence $n_{k}$,

$$
E \phi\left(u_{n_{k}}^{(R)}(, ; x)\right) \longrightarrow E \phi\left(u^{(R)}(, ; x)\right),
$$

for a.e. $t \in[0, T]$. Take $x, y \in H_{0}^{1}$, by the previous argument, we can find a subsequence $n_{k}$ such that the previous almost sure convergence in $t \in[0, T]$ holds true both $x$ and $y$.

Thus, from (212), we have

$$
\left|P_{t}^{(R)} \phi(x)-P_{t}^{(R)} \phi(y)\right| \leq L\|x-y\|_{H^{1}},
$$

for a.e. $t \in[0, T]$. As $u^{(R)}(t ; x)$ has continuous trajectories with values in $H_{0}^{1}$, the above inequality holds for all $t \in[0, T]$.

Proposition 14. Under conditions of Theorem 9, (S) holds true.

Proof. Take $t>0, x_{n}, x \in H_{0}^{1}$ satisfying $x_{n} \rightarrow x$ in $H^{1}$. For every $R>0$, we have that

$$
\begin{aligned}
& \left\|P_{R}\left(t, x_{n}, \cdot\right)-P_{R}(t, x, \cdot)\right\|_{T V} \\
& \quad=\sup _{\|\phi\|_{C_{b}\left(H^{1}\right)} \leq 1}\left|P_{t}^{(R)} \phi\left(x_{n}\right)-P_{t}^{(R)} \phi(x)\right| \\
& \quad \leq L\left\|x_{n}-x\right\|_{H^{1}} \longrightarrow 0
\end{aligned}
$$

as $n \rightarrow \infty$ by Proposition 13. Then,

$$
\begin{aligned}
\left\|P_{R}\left(t, x_{n}, \cdot\right)-P\left(t, x_{n}, \cdot\right)\right\|_{T V} \\
+\left\|P_{R}(t, x, \cdot)-P(t, x, \cdot)\right\|_{T V} \\
=\sup _{\|\phi\|_{C_{b}\left(H^{1}\right)} \leq 1}\left|P_{t}^{(R)} \phi\left(x_{n}\right)-P_{t} \phi\left(x_{n}\right)\right| \\
\quad+\sup _{\|\phi\|_{C_{b}\left(H^{1}\right)} \leq 1}\left|P_{t}^{(R)} \phi(x)-P_{t} \phi(x)\right| \\
=\sup _{\|\phi\|_{C_{b}\left(H^{1}\right)} \leq 1}\left|E \phi\left(u_{R}\left(t ; x_{n}\right)\right)-E \phi\left(u\left(t ; x_{n}\right)\right)\right| \\
\quad+\sup _{\|\phi\|_{C_{b}\left(H^{1}\right)} \leq 1}\left|E \phi\left(u_{R}(t ; x)\right)-E \phi(u(t ; x))\right| \\
\leq 2 \int_{\Omega} I_{\left\{\sup _{n \in \mathbb{N}}\left\|u\left(t ; x_{n}\right)\right\|_{H^{1}}>R\right\}} P(d w) \\
\quad+2 \int_{\Omega} I_{\left\{\|u(t ; x)\|_{H^{1}}>R\right\}} P(d w) \longrightarrow 0, \quad \text { as } R \rightarrow \infty,
\end{aligned}
$$

where the inequality follows by the consistency of $u(t ; x)$ and $u^{(R)}(t ; x)$, when $\|u(t ; x)\|_{H^{1}} \leq R$, and the limit follows by (A.21). Therefore,

$$
\begin{aligned}
&\left\|P\left(t, x_{n}, \cdot\right)-P(t, x, \cdot)\right\|_{T V} \\
& \leq\left\|P\left(t, x_{n}, \cdot\right)-P_{R}\left(t, x_{n}, \cdot\right)\right\|_{T V} \\
&+\left\|P_{R}\left(t, x_{n}, \cdot\right)-P_{R}(t, x, \cdot)\right\|_{T V} \\
&+\left\|P_{R}(t, x, \cdot)-P(t, x, \cdot)\right\|_{T V} \longrightarrow 0,
\end{aligned}
$$

as $n \rightarrow \infty$.

\section{Example}

Our theory can be applied to stochastic reaction diffusion equations or stochastic real valued Ginzburg Landau equation in high dimensions as follows:

$$
\begin{gathered}
\frac{\partial u}{\partial t}-\Delta u+|u|^{2} u-u=d W, \quad \text { on }[0, T] \times D, \\
u(t, x)=0, \quad t \in[0, T], x \in \partial D, \\
u(0, x)=u_{0}(x), \quad x \in D,
\end{gathered}
$$

where $u(t, x)=\left(u^{1}(t, x), u^{2}(t, x)\right)$ is the velocity field, $\Delta$ denotes the Laplace operator, $W$ stands for the $Q$-Wiener process, and $D$ is a regular bounded open domain of $\mathbb{R}^{2}$.

\section{Appendix}

Fix $R>0$ and let $K_{R}:\left[0, \infty\left[\rightarrow\left[0, \infty\left[\right.\right.\right.\right.$ satisfy $K_{R} \in C^{1}\left(\mathbb{R}_{+}\right)$ such that $\left|K_{R}\right| \leq 1,\left|K_{R}^{\prime}\right| \leq 2$ and

$$
\begin{gathered}
K_{R}(x)=1, \quad \text { if } x<R, \\
K_{R}(x)=0, \quad \text { if } x \geq R+1 .
\end{gathered}
$$


Consider the following equation:

$$
\begin{aligned}
& \frac{d v_{n}}{d t}+A v_{n}+K_{R}\left(\left\|v_{n}+P_{n} \phi\right\|_{H^{1}}^{2}\right) \\
& \quad \times P_{n} B\left(v_{n}+P_{n} \phi, v_{n}+P_{n} \phi\right) \\
& \quad+K_{R}\left(\left\|v_{n}+P_{n} \phi\right\|_{H^{1}}^{2}\right) f\left(v_{n}+P_{n} \phi\right)=0,
\end{aligned}
$$

where $\phi \in C\left([0, T] ; H^{3 / 2}\right)$.

Estimate 1. We have the following estimate in $H$ for (A.2):

$$
\left\|v_{n}\right\|_{C([0, T] ; H)}+\left\|v_{n}\right\|_{L^{2}\left([0, T] ; H^{1}\right)} \leq C\left(\|x\|_{H},\|\phi\|_{C\left([0, T] ; H^{3 / 2}\right)}, T\right),
$$

where $C(a, b, c)$ indicates a constant $C$ depending on $a, b, c$. Analogously to the derivation of (147), we get

$$
\frac{d}{d t}\left\|v_{n}\right\|_{H}^{2}+\left\|v_{n}\right\|_{H^{1}}^{2}+\left\|v_{n}\right\|_{L^{4}}^{4} \leq C\left(\|\phi\|_{H^{3 / 2}}^{4}+1\right)
$$

Therefore, for all $t \in[0, T]$,

$$
\begin{array}{r}
\left\|v_{n}(t)\right\|_{H}^{2}+\int_{0}^{t}\left\|v_{n}\right\|_{H^{1}}^{2} d s+\int_{0}^{t}\left\|v_{n}\right\|_{L^{4}}^{4} d s \\
\leq\|x\|_{H}^{2}+C \int_{0}^{t}\left(\|\phi(s)\|_{H^{3 / 2}}^{4}+1\right),
\end{array}
$$

Then, we get (A.3).

Estimate 2. We obtain the following estimate in $H_{0}^{1}$ for (A.2):

$$
\begin{aligned}
\| v_{n}(t) & \left\|_{C\left([0, T] ; H_{0}^{1}\right)}^{2}+\int_{0}^{T}\right\| v_{n}(s) \|_{H^{2}}^{2} d s \\
& \leq C\left(\|x\|_{H^{1}},\|\phi\|_{C\left([0, T] ; H^{3 / 2}\right)}, T\right) .
\end{aligned}
$$

Since we have

$$
\begin{aligned}
\frac{d}{d t}\left\|v_{n}(t)\right\|_{H^{1}}^{2}+\left\|v_{n}(t)\right\|_{H^{2}}^{2} \\
+\left\langle f\left(v_{n}(t)+P_{n} \phi(t)\right), A v_{n}(t)\right\rangle \\
=\left\langleB \left( v_{n}(t)+P_{n} \phi(t), v_{n}(t)\right.\right. \\
\left.\left.+P_{n} \phi(t)\right), A v_{n}(t)\right\rangle
\end{aligned}
$$

the equation is equivalent to

$$
\begin{aligned}
\frac{d}{d t}\left\|v_{n}(t)\right\|_{H^{1}}^{2}+\left\|v_{n}(t)\right\|_{H^{2}}^{2} \\
+\left\langle f\left(v_{n}(t)+P_{n} \phi(t)\right), A\left(v_{n}(t)+P_{n} \phi(t)\right)\right\rangle \\
=\left\langle B\left(v_{n}(t)+P_{n} \phi(t), v_{n}(t)+P_{n} \phi(t)\right), A v_{n}(t)\right\rangle \\
+\left\langle f\left(v_{n}(t)+P_{n} \phi(t)\right), A P_{n} \phi(t)\right\rangle .
\end{aligned}
$$

Denote by $u_{n}:=v_{n}(t)+P_{n} \phi(t)$ and $u_{n}=\left(u_{n}^{1}, u_{n}^{2}\right)$; then

$$
\begin{aligned}
\left\langle\left|u_{n}\right|^{2} u_{n}, A u_{n}\right\rangle= & 3 \int_{D}\left(u_{n}^{1}\right)^{2}\left(\partial_{1} u_{n}^{1}\right)^{2} d x \\
& +3 \int_{D}\left(u_{n}^{1}\right)^{2}\left(\partial_{2} u_{n}^{1}\right)^{2} d x \\
& +3 \int_{D}\left(u_{n}^{2}\right)^{2}\left(\partial_{1} u_{n}^{2}\right)^{2} d x \\
& +3 \int_{D}\left(u_{n}^{2}\right)^{2}\left(\partial_{2} u_{n}^{1}\right)^{2} d x \\
& +\int_{D}\left(u_{n}^{2}\right)^{2}\left(\partial_{1} u_{n}^{1}\right)^{2} d x \\
& +\int_{D}\left(u_{n}^{2}\right)^{2}\left(\partial_{2} u_{n}^{1}\right)^{2} d x \\
& +\int_{D}\left(u_{n}^{1}\right)^{2}\left(\partial_{1} u_{n}^{2}\right)^{2} d x \\
& +\int_{D}\left(u_{n}^{1}\right)^{2}\left(\partial_{2} u_{n}^{2}\right)^{2} d x \\
& +4 \int_{D}\left(u_{n}^{1} \partial_{1} u_{n}^{1}\right)\left(u_{n}^{2} \partial_{1} u_{n}^{2}\right) d x \\
& +4 \int_{D}\left(u_{n}^{1} \partial_{2} u_{n}^{1}\right)\left(u_{n}^{2} \partial_{2} u_{n}^{2}\right) d x
\end{aligned}
$$

As

$$
\begin{gathered}
4 \int_{D}\left(u_{n}^{1} \partial_{1} u_{n}^{1}\right)\left(u_{n}^{2} \partial_{1} u_{n}^{2}\right) d x \\
\leq 2 \int_{D}\left(u_{n}^{1}\right)^{2}\left(\partial_{1} u_{n}^{1}\right)^{2} d x \\
+2 \int_{D}\left(u_{n}^{2}\right)^{2}\left(\partial_{1} u_{n}^{2}\right)^{2} d x \\
4 \int_{D}\left(u_{n}^{1} \partial_{2} u_{n}^{1}\right)\left(u_{n}^{2} \partial_{2} u_{n}^{2}\right) d x \\
\leq 2 \int_{D}\left(u_{n}^{1}\right)^{2}\left(\partial_{2} u_{n}^{1}\right)^{2} d x \\
+2 \int_{D}\left(u_{n}^{2}\right)^{2}\left(\partial_{2} u_{n}^{2}\right)^{2} d x
\end{gathered}
$$

so, we have that

$$
\begin{aligned}
& \left\langle\left|u_{n}\right|^{2} u_{n}, A u_{n}\right\rangle \\
& \geq \int_{D}\left(u_{n}^{1}\right)^{2}\left(\partial_{1} u_{n}^{1}\right)^{2} d x \\
& \quad+\int_{D}\left(u_{n}^{1}\right)^{2}\left(\partial_{2} u_{n}^{1}\right)^{2} d x \\
& \quad+\int_{D}\left(u_{n}^{2}\right)^{2}\left(\partial_{1} u_{n}^{2}\right)^{2} d x \\
& \quad+\int_{D}\left(u_{n}^{2}\right)^{2}\left(\partial_{2} u_{n}^{2}\right)^{2} d x
\end{aligned}
$$




$$
\begin{aligned}
& +\int_{D}\left(u_{n}^{2}\right)^{2}\left(\partial_{1} u_{n}^{1}\right)^{2} d x \\
& +\int_{D}\left(u_{n}^{2}\right)^{2}\left(\partial_{2} u_{n}^{1}\right)^{2} d x \\
& +\int_{D}\left(u_{n}^{1}\right)^{2}\left(\partial_{1} u_{n}^{2}\right)^{2} d x \\
& +\int_{D}\left(u_{n}^{1}\right)^{2}\left(\partial_{2} u_{n}^{2}\right)^{2} d x \\
& =\int_{D}\left|u_{n}\right|^{2}\left|\nabla u_{n}\right|^{2} d x .
\end{aligned}
$$

For the first term on the right hand side of (A.3), we have

$$
\begin{aligned}
& \left\langle\left[\left(u_{n} \cdot \nabla\right) u_{n}\right], A v_{n}(t)\right\rangle \\
& \quad \leq\left\|v_{n}(t)\right\|_{H^{2}}^{2}+\frac{1}{4} \int_{D}\left\|u_{n}\right\|^{2}\left\|\nabla u_{n}\right\|^{2} d x .
\end{aligned}
$$

Substitute (A.11) and (A.12) into (A.8), we get

$$
\begin{aligned}
\frac{d}{d t} \| v_{n} & (t)\left\|_{H^{1}}^{2}+\right\| v_{n}(t) \|_{H^{2}}^{2} \\
& \leq\left\langle f\left(v_{n}(t)+P_{n} \phi(t)\right), A \phi(t)\right\rangle \\
& =\left\langle A^{1 / 4} f\left(v_{n}(t)+P_{n} \phi(t), A^{3 / 4} \phi(t)\right)\right\rangle .
\end{aligned}
$$

Denote

$$
u_{n}(t)=v_{n}(t)+P_{n} \phi(t) .
$$

Then,

$$
\begin{aligned}
\left\langle A^{1 / 4} f\left(v_{n}(t)+P_{n} \phi(t), A^{3 / 4} \phi(t)\right)\right\rangle & \\
\leq & \|\phi(t)\|_{H^{3 / 2}} \cdot\left\|A^{1 / 4}\left(\left|u_{n}(t)\right|^{2} u_{n}(t)\right)\right\|_{H} \\
\leq & \|\phi(t)\|_{H^{3 / 2}} \\
& \cdot\left\|\left(A^{1 / 4}\left|u_{n}(t)\right|^{2}\right) u_{n}(t)+\left|u_{n}(t)\right|^{2} A^{1 / 4} u_{n}(t)+R\right\|_{H} \\
\leq & \|\phi(t)\|_{H^{3 / 2}} \\
& {\left[\left\|\left(A^{1 / 4}\left|u_{n}(t)\right|^{2}\right) u_{n}(t)\right\|_{H}\right.} \\
& \left.\quad+\left\|\left.u_{n}(t)\right|^{2} A^{1 / 4} u_{n}(t)\right\|_{H}+\|R\|_{H}\right] \\
= & \|\phi(t)\|_{H^{3 / 2}} \cdot\left[I_{1}+I_{2}+I_{3}\right],
\end{aligned}
$$

where

$$
\begin{aligned}
R= & A^{1 / 4}\left(\left|u_{n}(t)\right|^{2} u_{n}(t)\right) \\
& -\left(A^{1 / 4}\left|u_{n}(t)\right|^{2}\right) u_{n}(t) \\
& -\left|u_{n}(t)\right|^{2} A^{1 / 4} u_{n}(t) .
\end{aligned}
$$

For $I_{1}$, we have

$$
I_{1} \leq\left\|\left(u_{n}(t) A^{1 / 4} u_{n}(t)+R_{1}\right) u_{n}(t)\right\|_{H}
$$

where

$$
R_{1}=A^{1 / 4}\left|u_{n}(t)\right|^{2}-2 u_{n}(t) A^{1 / 4} u_{n}(t) .
$$

So,

$$
\begin{aligned}
I_{1} \leq & C\left\|\left.u_{n}(t)\right|^{2} A^{1 / 4} u_{n}(t)+R_{1} u_{n}(t)\right\|_{H} \\
\leq & C\left\|u_{n}(t)\right\|_{L^{8}}^{2}\left\|u_{n}(t)\right\|_{H^{1 / 2,4}} \\
& +\left\|u_{n}(t)\right\|_{L^{4}}\left\|R_{1}\right\|_{L^{4}} \\
\leq & C\left\|u_{n}(t)\right\|_{L^{L^{\prime}}}^{2}\left\|u_{n}(t)\right\|_{H^{1 / 2,4}} \\
& +\left\|u_{n}(t)\right\|_{L^{4}}\left\|u_{n}(t)\right\|_{H^{1 / 4,8}} \\
\leq & C\left\|u_{n}(t)\right\|_{H^{1}}^{3} .
\end{aligned}
$$

Analogously, we can get the same estimate for $I_{2}$ and $I_{3}$. Take advantage of the estimates for $I_{1}, I_{2}$, and $I_{3}$, we have

$$
\begin{aligned}
& \frac{d}{d t}\left\|v_{n}(t)\right\|_{H^{1}}^{2}+\left\|v_{n}(t)\right\|_{H^{2}}^{2} \\
& \quad \leq C\|\phi(t)\|_{H^{3 / 2}}\left\|u_{n}(t)\right\|_{H^{1}}^{3} \\
& \quad \leq C\left(\left\|v_{n}(t)\right\|_{H^{1}}^{3}+\|\phi(t)\|_{H^{3 / 2}}^{3}\right) .
\end{aligned}
$$

By the Gronwall inequality and (A.3), we get (A.6).

Remark A.1. It is standard to show that, for $x \in H_{0}^{1}$ and $\phi \epsilon$ $C\left([0, T] ; H^{3 / 2}\right)$, there exists a subsequence which converges to some $v$, strongly in $L^{2}\left([0, T] ; H^{1}\right)$, weekly in $L^{2}\left([0, T] ; H^{2}\right)$, and weak star in $L^{\infty}\left([0, T] ; H^{1}\right)$. Therefore, we have

$$
\begin{aligned}
& \|v(t)\|_{C\left([0, T] ; H_{0}^{1}\right)}^{2}+\int_{0}^{T}\|v(s)\|_{H^{2}}^{2} d s \\
& \quad \leq C\left(\|x\|_{H^{1}},\|\phi\|_{C\left([0, T] ; H^{3 / 2}\right)}, T\right) .
\end{aligned}
$$

Estimate 3. We compare, only in the case $R=\infty$. Let $v_{n}^{1}, v_{n}^{2}$ be two solutions with the same initial condition $x \in H^{1}$ but with different functions $\phi_{1}, \phi_{2}$, there exists a constant $C\left(\|x\|_{H^{1}},\left\|\phi_{1}\right\|_{C\left([0, T] ; H^{3 / 2}\right)},\left\|\phi_{2}\right\|_{C\left([0, T] ; H^{3 / 2}\right)}, T\right)$, such that

$$
\begin{aligned}
& \left\|v_{n}^{1}-v_{n}^{2}\right\|_{C\left([0, T] ; H_{0}^{1}\right)} \\
& \leq C\left(\|x\|_{H^{1}},\left\|\phi_{1}\right\|_{C\left([0, T] ; H^{3 / 2}\right)},\left\|\phi_{2}\right\|_{C\left([0, T] ; H^{3 / 2}\right)}, T\right) \\
& \quad \times\left\|\phi_{1}-\phi_{2}\right\|_{C\left([0, T] ; H^{3 / 2}\right)},
\end{aligned}
$$

for every $n, x \in H^{1}, \phi_{1}, \phi_{2}, T$. We have

$$
\begin{aligned}
& \frac{d v_{n}^{i}}{d t}+A v_{n}^{i}+P_{n} B\left(v_{n}^{i}+P_{n} \phi_{i}, v_{n}^{i}+P_{n} \phi_{i}\right) \\
& +\vartheta\left|v_{n}^{i}+P_{n} \phi_{i}\right|^{2}\left(v_{n}^{i}+P_{n} \phi_{i}\right)=0,
\end{aligned}
$$


with initial condition $v_{n}^{i}(0)=P_{n} x$, for $i=1,2$. Set $\eta_{n}=v_{n}^{1}-$ $v_{n}^{2}, \psi=\phi_{1}-\phi_{2}$. Then,

$$
\begin{aligned}
& \frac{d \eta_{n}}{d t}+A \eta_{n}+P_{n} B\left(v_{n}^{1}+P_{n} \phi_{1}, \eta_{n}+P_{n} \psi\right) \\
& \quad+P_{n} B\left(\eta_{n}+P_{n} \psi, v_{n}^{2}+P_{n} \phi_{2}\right) \\
& \quad+\vartheta\left|v_{n}^{1}+\phi_{1}\right|^{2}\left(v_{n}^{1}+\phi_{1}\right) \\
& \quad-\vartheta\left|v_{n}^{2}+\phi_{2}\right|^{2}\left(v_{n}^{2}+\phi_{2}\right)=0 .
\end{aligned}
$$

Take inner product in $H$ with respect to $A \eta_{n}$, we have

$$
\begin{gathered}
\frac{1}{2} \frac{d}{d t}\left\|\eta_{n}\right\|_{H^{1}}^{2}+\left\|\eta_{n}\right\|_{H^{2}}^{2} \\
+\left\langle P_{n} B\left(\left(v_{n}^{1}+P_{n} \phi_{1}\right),\left(\eta_{n}+P_{n} \psi\right)\right), A \eta_{n}\right\rangle \\
+\left\langle P_{n} B\left(\eta_{n}+P_{n} \psi, v_{n}^{2}+P_{n} \phi_{2}\right), A \eta_{n}\right\rangle \\
+\vartheta\left\langle\left|v_{n}^{1}+\phi_{1}\right|^{2}\left(v_{n}^{1}+\phi_{1}\right)\right. \\
\left.\quad-\left|v_{n}^{2}+\phi_{2}\right|^{2}\left(v_{n}^{2}+\phi_{2}\right), A \eta_{n}\right\rangle=0 .
\end{gathered}
$$

For the third term on the left hand side of (A.23), we have

$$
\begin{aligned}
& \left\langle P_{n} B\left(v_{n}^{1}+P_{n} \phi_{1}, \eta_{n}+P_{n} \psi\right), A \eta_{n}\right\rangle \\
& \leq\left\|\eta_{n}\right\|_{H^{2}}\left\|\eta_{n}+P_{n} \psi\right\|_{H^{1,4}}\left\|v_{n}^{1}+P_{n} \phi_{1}\right\|_{L^{4}} \\
& \leq\left\|\eta_{n}\right\|_{H^{2}}\left(\left\|\eta_{n}\right\|_{H^{3 / 2}}+\|\psi\|_{H^{3 / 2}}\right)\left(\left\|v_{n}^{1}+\phi_{1}\right\|_{H^{1}}\right) \\
& \leq\left\|v_{n}^{1}+\phi_{1}\right\|_{H^{1}}\left\|\eta_{n}\right\|_{H^{2}}\left(\left\|\eta_{n}\right\|_{H^{1}}^{1 / 2}\left\|\eta_{n}\right\|_{H^{2}}^{1 / 2}+\|\psi\|_{H^{3 / 2}}\right) \\
& \leq \varepsilon\left\|\eta_{n}\right\|_{H^{2}}^{2}+C\left\|v_{n}^{1}+\phi_{1}\right\|_{H^{1}}^{4}\left\|\eta_{n}\right\|_{H^{1}}^{2} \\
& \quad+C\left\|v_{n}^{1}+\phi_{1}\right\|_{H^{1}}^{2}\|\psi\|_{H^{3 / 2}}^{2} .
\end{aligned}
$$

$$
\begin{aligned}
&\left\langle P_{n} B\left(\eta_{n}+P_{n} \psi, v_{n}^{2}+P_{n} \phi_{2}\right), A \eta_{n}\right\rangle \\
& \leq \varepsilon\left\|\eta_{n}\right\|_{H^{2}}^{2}+C\left\|v_{n}^{2}\right\|_{H^{2}}^{2}\left\|\eta_{n}\right\|_{H^{1}}^{2} \\
&+C\left\|v_{n}^{2}\right\|_{H^{2}}^{2}\|\psi\|_{H^{1}}^{2} \\
&+C\left\|\phi_{2}\right\|_{H^{3 / 2}}^{2}\left\|\eta_{n}\right\|_{H^{1}}^{2} \\
&+C\left\|\phi_{2}\right\|_{H^{3 / 2}}^{2}\|\psi\|_{H^{1}}^{2}, \\
& \vartheta\langle| v_{n}^{1}+\left.\left.\phi_{1}\right|^{2}\left(v_{n}^{1}+\phi_{1}\right)-\left|v_{n}^{2}+\phi_{2}\right|^{2}\left(v_{n}^{2}+\phi_{2}\right), A \eta_{n}\right\rangle \\
& \leq \varepsilon\left\|\eta_{n}\right\|_{H^{2}}^{2}+C\left\|\eta_{n}\right\|_{H^{1}}^{2}\left(\left\|v_{n}^{1}+\phi_{1}\right\|_{H^{1}}^{4}+\left\|v_{n}^{2}+\phi_{2}\right\|_{H^{1}}^{4}\right) \\
&+C\|\psi\|_{H^{1}}^{2}\left(\left\|v_{n}^{1}+\phi_{1}\right\|_{H^{1}}^{4}+\left\|v_{n}^{2}+\phi_{2}\right\|_{H^{1}}^{4}\right) .
\end{aligned}
$$$$
\text { we can get }
$$

By (A.23)-(A.27), we have

$$
\begin{gathered}
\frac{d}{d t}\left\|\eta_{n}\right\|_{H^{1}}^{2}+\left\|\eta_{n}\right\|_{H^{2}}^{2} \\
\leq C\left\|\eta_{n}\right\|_{H^{1}}^{2}\left(\left\|v_{n}^{1}+\phi_{1}\right\|_{H^{1}}^{4}+\left\|v_{n}^{2}+\phi_{2}\right\|_{H^{1}}^{4}\right. \\
\left.+\left\|v_{n}^{1}+\phi_{1}\right\|_{H^{1}}^{2}+\left\|v_{n}^{2}\right\|_{H^{2}}^{2}+\left\|\phi_{2}\right\|_{H^{3 / 2}}^{2}\right) \\
+C\|\psi\|_{H^{3 / 2}}^{2}\left(\left\|v_{n}^{1}+\phi_{1}\right\|_{H^{1}}^{4}+\left\|v_{n}^{2}+\phi_{2}\right\|_{H^{1}}^{4}\right. \\
\left.+\left\|v_{n}^{1}+\phi_{1}\right\|_{H^{1}}^{2}+\left\|v_{n}^{2}\right\|_{H^{2}}^{2}+\left\|\phi_{2}\right\|_{H^{3 / 2}}^{2}\right) .
\end{gathered}
$$

So, by the Gronwall inequality and (A.6), we get (A.21). By (A.6), we know that $v_{n}^{i}$ converges week star to $v^{i}$ in $C\left([0, T] ; H_{0}^{1}\right)$, for $i=1,2$, we have

$$
\begin{aligned}
& \left\|v^{1}-v^{2}\right\|_{C\left([0, T] ; H^{1}\right)} \\
& \leq C\left(\|x\|_{H^{1}},\left\|\phi_{1}\right\|_{C\left([0, T] ; H^{3 / 2}\right)},\left\|\phi_{2}\right\|_{C\left([0, T] ; H^{3 / 2}\right)}, T\right) \\
& \quad\left\|\phi_{1}-\phi_{2}\right\|_{C\left([0, T] ; H^{3 / 2}\right)} .
\end{aligned}
$$

Estimate 4. Let us consider only the case $R \in(0, \infty)$, and denote by $v_{n}(t)$ the solution to (A.2). Let $\xi_{n}$ be the differential mapping $x \rightarrow v_{n}$ in the direction $h$ at point $x$, defined by, for given $x, h \in H$ as follows:

$$
\xi_{n}(t)=D_{x} v_{n}(t ; x) \cdot h
$$

Set also

$$
u_{n}(t ; x)=v_{n}(t, x)+P_{n} \phi(t),
$$

so that $\xi_{n}$ is also the differential of the mapping $x \rightarrow u_{n}(t ; x)$ in the direction $h$ at the point $x$. Thus, $\xi_{n}$ satisfies

$$
\begin{aligned}
\frac{d}{d t} \xi_{n}+ & A \xi_{n} \\
= & 2 K_{R}^{\prime}\left(\left\|u_{n}\right\|_{H^{1}}^{2}\right)\left\langle A^{1 / 2} u_{n}, A^{1 / 2} \xi_{n}\right\rangle B\left(u_{n}, u_{n}\right) \\
& +K_{R}\left(\left\|u_{n}\right\|_{H^{1}}^{2}\right)\left\{B\left(u_{n}, \xi_{n}\right)+B\left(\xi_{n}, u_{n}\right)\right\} \\
& +2 K_{R}^{\prime}\left(\left\|u_{n}\right\|_{H^{1}}^{2}\right)\left\langle A^{1 / 2} u_{n}, A^{1 / 2} \xi_{n}\right\rangle u_{n}^{3} \\
& +3 K_{R}\left(\left\|u_{n}\right\|_{H^{1}}^{2}\right)\left|u_{n}\right|^{2} \xi_{n} .
\end{aligned}
$$

So,

$$
\begin{aligned}
\frac{d}{d t}\left\|\xi_{n}\right\|_{H^{1}}^{2}+\left\|\xi_{n}\right\|_{H^{2}}^{2} \\
=2 K_{R}^{\prime}\left(\left\|u_{n}\right\|_{H}^{2}\right)\left\langle B\left(u_{n}, u_{n}\right), A \xi_{n}\right\rangle \\
+K_{R}\left(\left\|u_{n}\right\|_{H}^{2}\right)\left\langle B\left(u_{n}, \xi_{n}\right), A \xi_{n}\right\rangle \\
+K_{R}\left(\left\|u_{n}\right\|_{H}^{2}\right)\left\langle B\left(\xi_{n}, u_{n}\right), A \xi_{n}\right\rangle \\
+2 K_{R}^{\prime}\left(\left\|u_{n}\right\|_{H}^{2}\right)\left\langle A^{1 / 2} u_{n}, A^{1 / 2} \xi_{n}\right\rangle\left\langle\left|u_{n}\right|^{2} u_{n}, A \xi_{n}\right\rangle \\
+3 K_{R}\left(\left\|u_{n}\right\|_{H}^{2}\right)\left\langle\left|u_{n}\right|^{2} \xi_{n}, A \xi_{n}\right\rangle .
\end{aligned}
$$


Therefore,

$$
\begin{aligned}
& \frac{d}{d t}\left\|\xi_{n}\right\|_{H^{1}}^{2}+\left\|\xi_{n}\right\|_{H^{2}}^{2} \\
& \leq 2 K_{R}^{\prime}\left(\left\|u_{n}\right\|_{H}^{2}\right)\left\|u_{n}\right\|_{H^{1}}\left\|\xi_{n}\right\|_{H^{1}}\left\|\xi_{n}\right\|_{H^{2}}\left\|u_{n}\right\|_{L^{4}}\left\|u_{n}\right\|_{H^{1,4}} \\
& \quad+K_{R}\left(\left\|u_{n}\right\|_{H^{1}}^{2}\right)\left\|\xi_{n}\right\|_{H^{2}}\left\|\xi_{n}\right\|_{H^{1,4}}\left\|u_{n}\right\|_{L^{4}} \\
& \quad+K_{R}\left(\left\|u_{n}\right\|_{H^{1}}^{2}\right)\left\|\xi_{n}\right\|_{H^{2}}\left\|\xi_{n}\right\|_{L^{4}}\left\|u_{n}\right\|_{H^{1,4}} \\
& \quad+2 K_{R}^{\prime}\left(\left\|u_{n}\right\|_{H^{1}}^{2}\right)\left\|u_{n}\right\|_{H^{1}}\left\|\xi_{n}\right\|_{H^{1}}\left\|\xi_{n}\right\|_{H^{2}}\left\|u_{n}\right\|_{L^{6}}^{3} \\
& \quad+3 K_{R}\left(\left\|u_{n}\right\|_{H^{1}}^{2}\right)\left\|\xi_{n}\right\|_{H^{2}}\left\|\xi_{n}\right\|_{L^{4}}\left\|u_{n}\right\|_{L^{8}}^{2} \\
& \leq C(R)\left\|\xi_{n}\right\|_{H^{1}}\left\|\xi_{n}\right\|_{H^{2}}\left(\left\|v_{n}\right\|_{H^{2}}+\|\phi\|_{H^{3 / 2}}\right) \\
& \quad+C(R)\left\|\xi_{n}\right\|_{H^{2}}^{3 / 2}\left\|\xi_{n}\right\|_{H^{1}}^{1 / 2} \\
& \quad+C(R)\left\|\xi_{n}\right\|_{H^{2}}\left\|\xi_{n}\right\|_{H^{1}} \\
& \leq \varepsilon\left\|\xi_{n}\right\|_{H^{2}}^{2}+C(R)\left\|\xi_{n}\right\|_{H^{1}}^{2} \\
& \quad \times\left(1+\left\|v_{n}\right\|_{H^{2}}^{2}+\|\phi\|_{H^{3 / 2}}^{2}\right) .
\end{aligned}
$$

By the Gronwall inequality and (A.6), we have

$$
\left\|\xi_{n}(t)\right\|_{H^{1}}^{2} \leq C(R)\|h\|_{H^{1}}^{2}
$$

And therefore, using again the previous inequality,

$$
\int_{0}^{T}\left\|\xi_{n}(t)\right\|_{H^{2}}^{2} d t \leq C(R)\|h\|_{H^{1}}^{2} .
$$

\section{Acknowledgments}

The authors are thankful to the referee for careful reading and insightful comments which led to many improvements of the earlier version. This work was partially supported by the Fundamental Research Funds for the Central Universities (Grant no. CQDXWL-2013-003).

\section{References}

[1] H. Beteman, "Some recent researches of the motion of fluid," Monthly Weather Review, vol. 43, pp. 163-170, 1915.

[2] A. R. Forsyth, Theory of Differential Equations, vol. 6, Cambridge University Press, Cambridge, UK, 1906.

[3] D. H. Chambers, R. J. Adrian, P. Moin, D. S. Stewart, and H. J. Sung, "Karhunen Loeve expansion of Burgers' model of turbulence," Physics of Fluids, vol. 31, pp. 2573-2582, 1988.

[4] H. C. Choi, R. Temam, P. Moin, and J. Kim, "Feedback control for unsteady flow and its application to the stochastic Burgers equation," Journal of Fluid Mechanics, vol. 253, pp. 509-543, 1993.

[5] D. Jeng, "Forced model equation for turbulence," The Physics of Fluids, vol. 12, no. 10, pp. 2006-2010, 1969.

[6] I. Hosokawa and K. Yamamoto, "Turbulence in the randomly forced, one-dimensional Burgers flow," Journal of Statistical Physics, vol. 13, no. 3, pp. 245-272, 1975.
[7] M. Kardar, G. Parisi, and Y. Zhang, "Dynamic scaling of growing interfaces," Physical Review Letters, vol. 56, no. 9, pp. 889-892, 1986.

[8] L. Bertini, N. Cancrini, and G. Jona-Lasinio, "The stochastic Burgers equation," Communications in Mathematical Physics, vol. 165, no. 2, pp. 211-232, 1994.

[9] G. Da Prato, A. Debussche, and R. Temam, "Stochastic Burgers' equation," Nonlinear Differential Equations and Applications, vol. 1, no. 4, pp. 389-402, 1994.

[10] G. Da Prato and A. Debussche, "Stochastic Cahn-Hilliard equation," Nonlinear Analysis: Theory, Methods \& Applications A, vol. 26, no. 2, pp. 241-263, 1996.

[11] I. Gyöngy and D. Nualart, "On the stochastic Burgers' equation in the real line," The Annals of Probability, vol. 27, no. 2, pp. 782802, 1999.

[12] M. Gourcy, "Large deviation principle of occupation measure for stochastic Burgers equation," Annales de l'Institut Henri Poincaré, vol. 43, no. 4, pp. 441-459, 2007.

[13] E. Weinan, K. Khanin, A. Mazel, and Y. Sinai, "Invariant measures for Burgers equation with stochastic forcing," Annals of Mathematics, vol. 151, no. 3, pp. 877-960, 2000.

[14] Z. Dong and T. G. Xu, "One-dimensional stochastic Burgers equation driven by Lévy processes," Journal of Functional Analysis, vol. 243, no. 2, pp. 631-678, 2007.

[15] G. Wang, M. Zeng, and B. Guo, 'Stochastic Burgers' equation driven by fractional Brownian motion," Journal of Mathematical Analysis and Applications, vol. 371, no. 1, pp. 210-222, 2010.

[16] Z. Hou, J. Luo, P. Shi, and S. K. Nguang, "Stochastic stability of Ito differential equations with semi-Markovian jump parameters," IEEE Transactions on Automatic Control, vol. 51, no. 8, pp. 1382-1386, 2006.

[17] F. Li, L. Wu, and P. Shi, "Stochastic stability of semi-Markovian jump systems with mode-dependent delays," International Journal of Robust and Nonlinear Control, 2013.

[18] J. Qiu, G. Feng, and J. Yang, "A new design of delay-dependent robust $H_{\infty}$ filtering for discrete-time T-S fuzzy systems with time-varying delay," IEEE Transactions on Fuzzy Systems, vol. 17, no. 5, pp. 1044-1058, 2009.

[19] J. Qiu, G. Feng, and J. Yang, "Delay-dependent nonsynchronized robust $H_{\infty}$ state estimation for discrete-time piecewise linear delay systems," International Journal of Adaptive Control and Signal Processing, vol. 23, no. 12, pp. 1082-1096, 2009.

[20] Y. Wei, J. Qiu, H. R. Karimi, and M. Wang, "A new design of $H_{\infty}$ filtering for continuous-time Markovian jump systems with time-varying delay and partially accessible mode information," Signal Processing, vol. 93, no. 9, pp. 2392-2407, 2013.

[21] M. Hairer, J. C. Mattingly, and M. Scheutzow, "Asymptotic coupling and a general form of Harris' theorem with applications to stochastic delay equations," Probability Theory and Related Fields, vol. 149, no. 1-2, pp. 223-259, 2011.

[22] J. L. Doob, "Asymptotic properties of Markoff transition prababilities," Transactions of the American Mathematical Society, vol. 64, pp. 393-421, 1948.

[23] G. Da Prato and J. Zabczyk, Stochastic Equations in Infinite Dimensions, Encyclopedia of Mathematics and its Applications, Cambridge University Press, Cambridge, UK, 1992.

[24] M. Rockner, Intruduction to Stochastic Partial Difficult Equation, Fall 2005 and Spring 2006, Version, Purdue University, 2007. 
[25] C. E. Kenig, G. Ponce, and L. Vega, "Well-posedness and scattering results for the generalized Korteweg-de Vries equation via the contraction principle," Communications on Pure and Applied Mathematics, vol. 46, no. 4, pp. 527-620, 1993.

[26] F. Flandoli, "Dissipativity and invariant measures for stochastic Navier-Stokes equations," Nonlinear Differential Equations and Applications, vol. 1, no. 4, pp. 403-423, 1994.

[27] A. Pazy, Semigroups of Linear Operators and Applications to Partial Differential Equations, Springer, New York, NY, USA, 1983.

[28] G. Da Prato and J. Zabczyk, Ergodicity for Infinite-Dimensional Systems, vol. 229 of London Mathematical Society Lecture Note Series, Cambridge University Press, Cambridge, UK, 1996.

[29] S. Pezat and J. Zabczyk, Strong Feller Property and Irreducibility for Diffusions on Hilbert Spaces, no. 510, Institute of Mathematics Polish Academy of Sciences, Warsaw, Poland, 1993. 


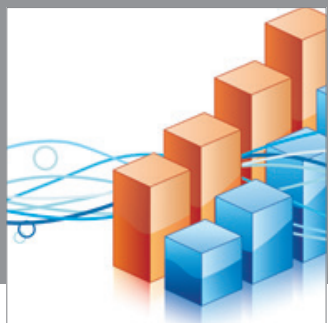

Advances in

Operations Research

mansans

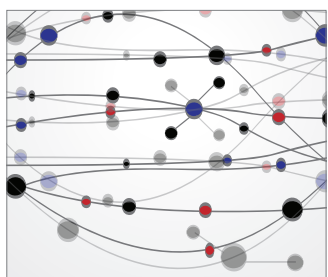

The Scientific World Journal
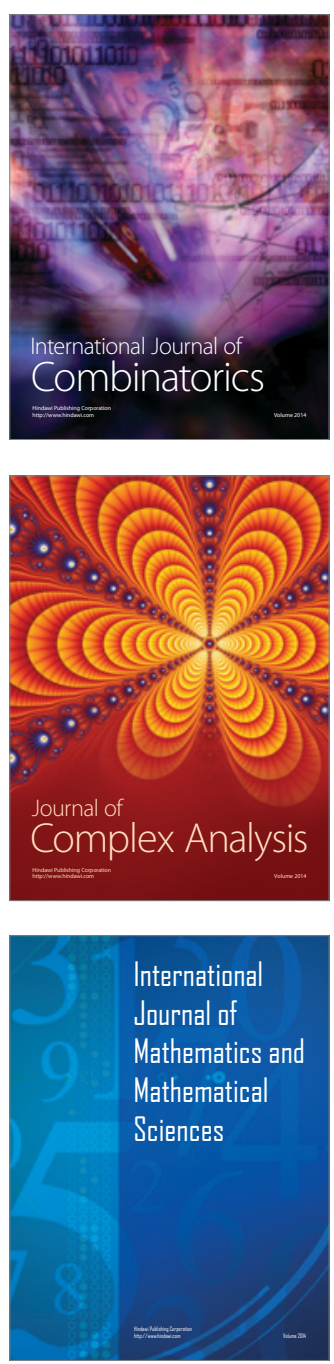
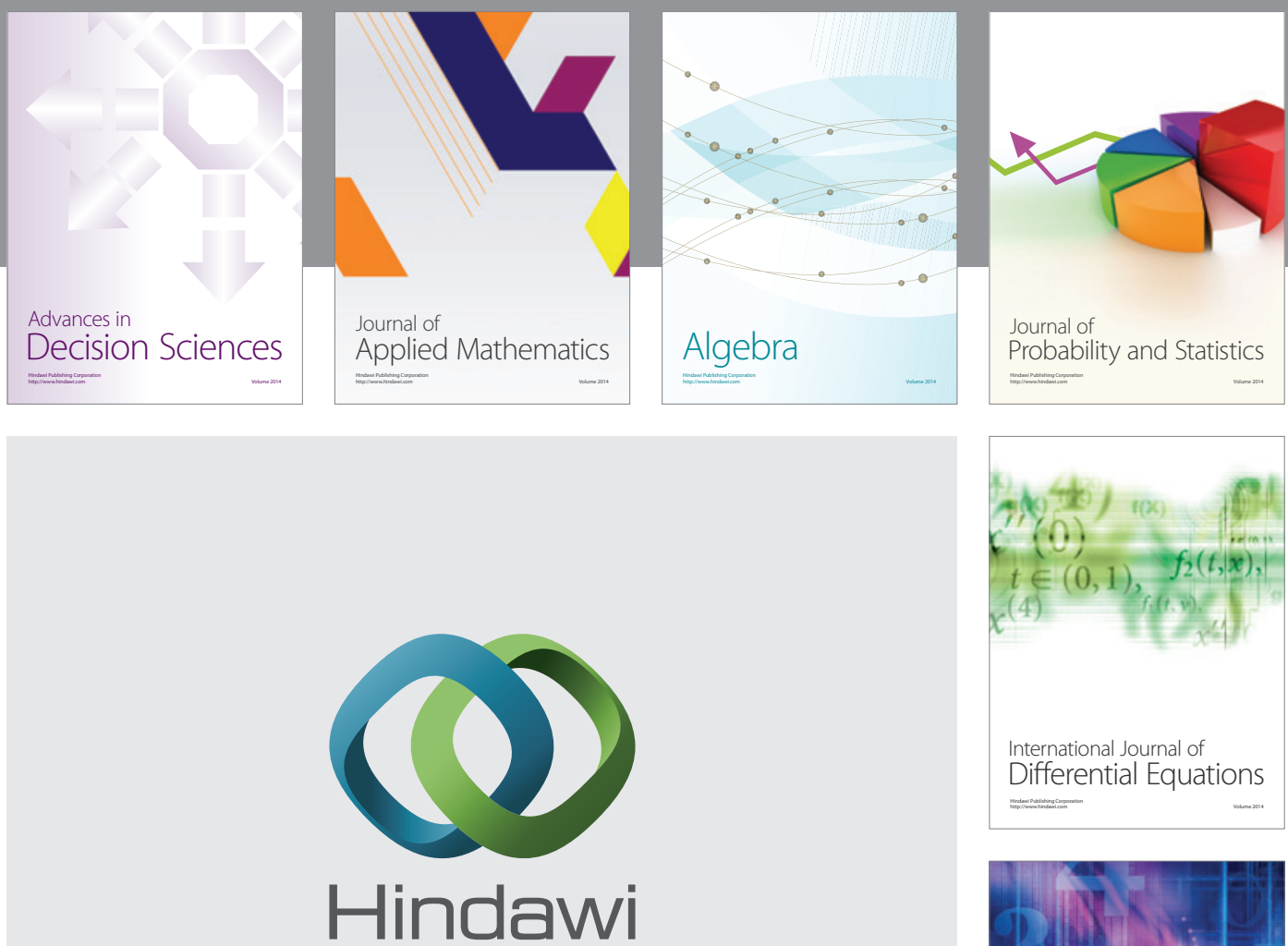

Submit your manuscripts at http://www.hindawi.com
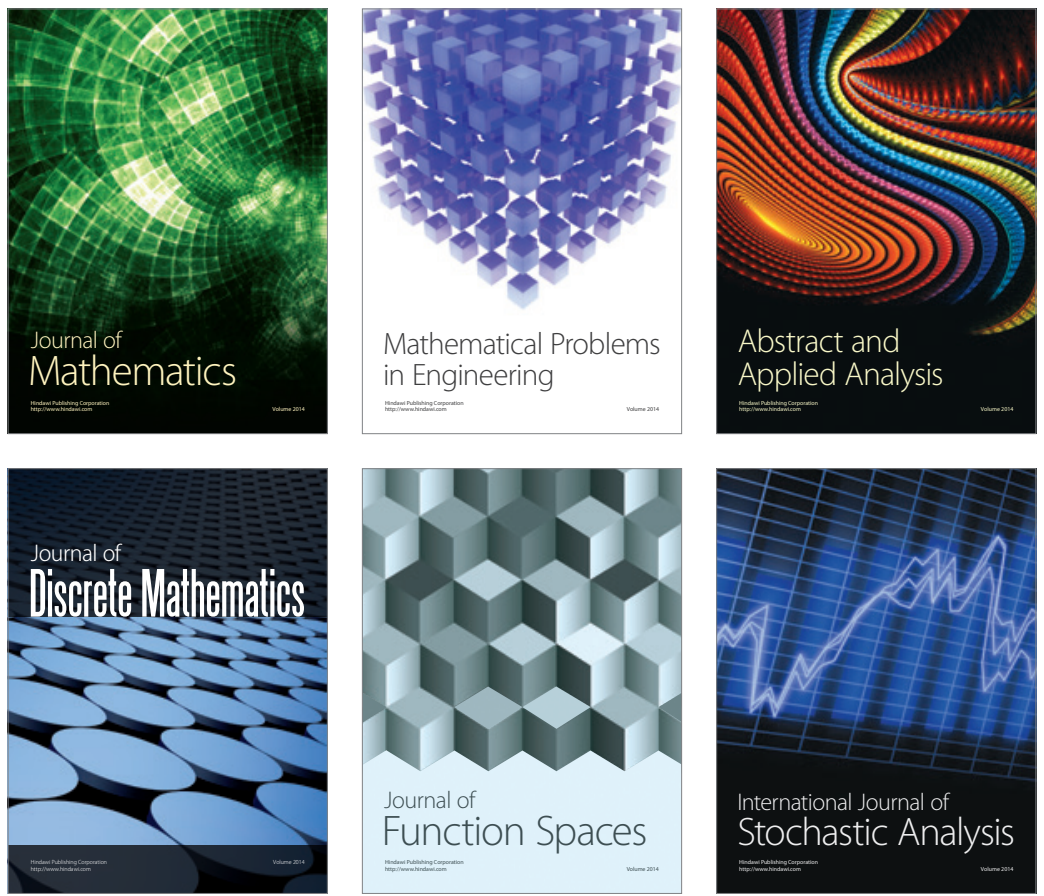

Journal of

Function Spaces

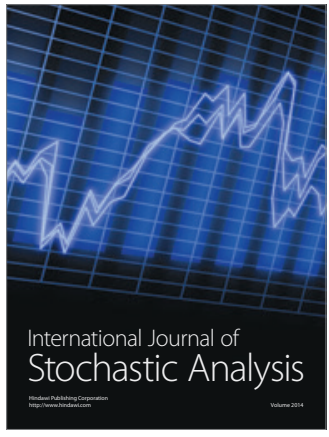

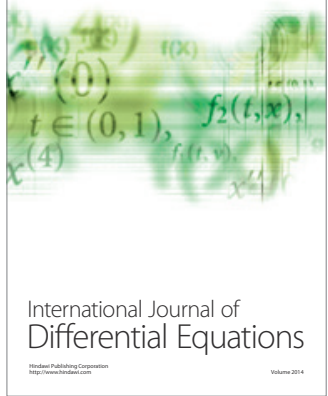
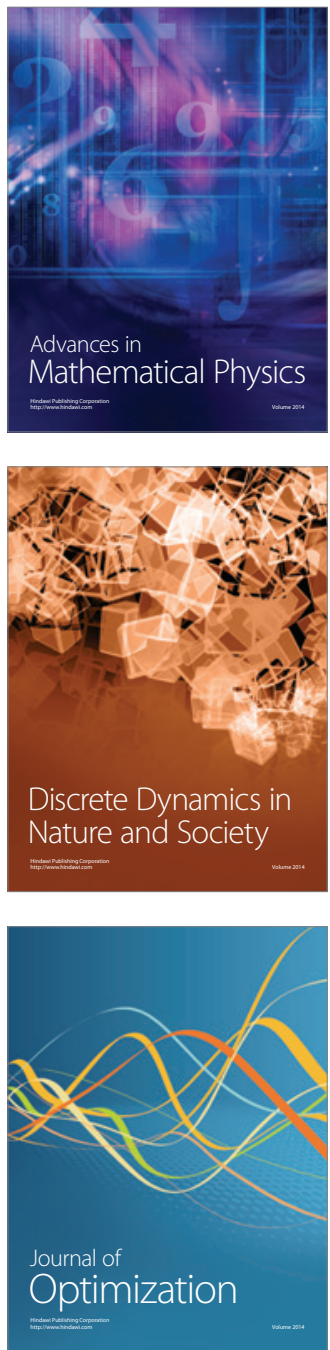